\title{
Path-dependent backward stochastic Volterra integral equations with jumps, differentiability and duality principle
}

\author{
Ludger Overbeck • Jasmin A. L. Röder (1)
}

Received: 30 May 2017 / Accepted: 23 April 2018 / Published online: 05 June 2018

(C) The Author(s). 2018 Open Access This article is distributed under the terms of the Creative Commons Attribution 4.0 International License (http://creativecommons.org/licenses/by/4.0/), which permits unrestricted use, distribution, and reproduction in any medium, provided you give appropriate credit to the original author(s) and the source, provide a link to the Creative Commons license, and indicate if changes were made.

\begin{abstract}
We study the existence and uniqueness of a solution to path-dependent backward stochastic Volterra integral equations (BSVIEs) with jumps, where pathdependence means the dependence of the free term and generator of a path of a càdlàg process. Furthermore, we prove path-differentiability of such a solution and establish the duality principle between a linear path-dependent forward stochastic Volterra integral equation (FSVIE) with jumps and a linear path-dependent BSVIE with jumps. As a result of the duality principle we get a comparison theorem and derive a class of dynamic coherent risk measures based on path-dependent BSVIEs with jumps.
\end{abstract}

Keywords Path-dependent backward stochastic Volterra integral equation · Jump diffusion · Path-differentiability · Duality principle $\cdot$ Comparison theorem . Functional Itô formula $\cdot$ Dynamic coherent risk measure

\section{Introduction}

Since Pardoux and Peng provided in Pardoux and Peng (1990) the existence and uniqueness to a non-linear backward stochastic differential equation (BSDE) with Lipschitz continuous generator and measurable terminal condition, this type of equation has attracted enormous attention in probability theory and its applications. Their research has been extended in terms of BSDEs with Poisson jumps by Tang

\footnotetext{
J. A. L. Röder (四)

Institute of Mathematics, University of Gießen, Arndtsraße 2, 35392 Gießen, Germany

e-mail: jasmin.roeder@math.uni-giessen.de

L. Overbeck

Institute of Mathematics, University of Gießen, Arndtsraße 2, 35392 Gießen, Germany

e-mail: ludger.overbeck@math.uni-giessen.de
} 
and Li (1994) and Barles et al. (1997) and for BSDEs driven by random measures by Becherer (2006). Moreover, path-dependent BSDEs with jumps, where the generator and the terminal condition both depend on a path of a càdlàg process, were studied by Wang (2015) and Kromer et al. (2017). BSDEs are important because of their connections to financial mathematics as studied in El Karoui et al. (1997), Rong (1997), Carmona (2008), Delong (2013), Crépey (2013), and many others.

Furthermore, BSDEs are special cases of backward stochastic Volterra integral equations (BSVIEs). Stochastic Volterra integral equations were first investigated by Berger and Mizel (1980) and extended by Protter (1985) and Pardoux and Protter (1990). Lin (2002) studied these equations in an $L^{2}$-framework under global Lipschitz conditions on the coefficients the existence and uniqueness of a solution to backward stochastic nonlinear Volterra integral equations of the form

$Y(t)=\xi+\int_{t}^{T} f(t, s, Y(s), Z(t, s)) d s+\int_{t}^{T}[g(t, s, Y(s))+Z(t, s)] d W(s), t \in[0, T]$.

This result was extended by Aman and N'Zi (2005) by weakening the global Lipschitz condition to a local one. Wang and Zhang (2007) proved existence and uniqueness of a solution to BSVIEs with jumps and non-Lipschitz coefficients. Moreover, they studied the regularity of this solution. A preliminary theory for BSVIEs was established by Yong (2006) considering a BSVIE of the form

$Y(t)=f(t)-\int_{t}^{T} h(t, s, Y(s), Z(t, s), Z(s, t)) d s-\int_{t}^{T} Z(t, s) d W(s), \quad t \in[0, T]$.

These results were extended by Anh and Yong (2006) and Yong (2008), where they considered the notion of adapted $M$-solutions and established the well-posedness of adapted $M$-solutions to BSVIEs. Furthermore, Yong (2006) and Wang and Yong (2015) introduced the duality principle between linear forward stochastic Volterra integral equations (FSVIEs) and linear BSVIEs to prove the comparison theorem. Their study was motivated firstly by the use of BSVIEs in the context of dynamic risk measures, as proposed by Yong (2007), and secondly by the connection between BSVIEs and stochastic differential utilities as introduced and studied in Duffie and Epstein (1992) and Lazrak and Quenez (2003).

Further, Ren (2010) studied the existence and uniqueness of a solution to BSVIEs driven by a cylindrical Brownian motion on a separable Hilbert space and a Poisson random measure with a non-Lipschitz coefficient. Moreover, he proved a duality principle between linear FSVIEs with jumps and linear BSVIEs with jumps.

The aim of this work is to study path-dependent BSVIEs with jumps, where the path-dependence means the dependence of the free term and the generator on a path of a càdlàg process. These types of path-dependent stochastic equations came into the focus of stochastic analysis, particularly since the development of a path-dependent Itô formula as in Dupire (2009), Cont and Fournié (2010), and Levental et al. (2013). It was first considered for backward equations by Peng and Wang in Peng and Wang (2016) and developed in Wang (2015). They also showed differentiability of path-dependent BSDEs in the Dupire sense and applied it to path-dependent partial differential equations. We extend their differentiability result to the BSVIE case. The 
Differentiability of BSDEs and BSVIEs was considered in a different context, e.g., Ankirchner et al. (2007) and Kromer and Overbeck (2014); Kromer and Overbeck (2017); Kromer et al. (2017). One application of BSVIEs is the risk measures associated with them. Path-dependency can then be incorporated to exhibit the dependency of the risk measures and the underlying portfolio from a driving exogenous factor process or asset price process $X$. Differentiability can then be used to measure the sensitivity of the risk with respect to this driving factor process. In Kromer and Overbeck (2014); Kromer and Overbeck (2017) a different kind of differentiability was used for the purpose of capital allocation for risk measures.

We derive an existence and uniqueness result for adapted $M$-solutions to pathdependent BSVIEs with jumps. Furthermore, we prove path-differentiability of this solution, where we use the functional Itô formula introduced by Dupire (2009) and extended by Cont and Fournié (2010) and Levental et al. (2013). Moreover, we establish a duality principle between linear path-dependent FSVIEs with jumps and linear path-dependent BSVIEs with jumps. The main challenge of our work is to handle the dependence of the generator and terminal condition of the path of a càdlàg process in the differentiability result, duality principle, and comparison theorem for path-dependent BSVIEs with jumps.

This work is organized as follows. In Section 2, we present necessary notations, spaces, and results to path-dependent BSDEs with jumps. Furthermore, we introduce the considered path-dependent BSVIE with jumps. In Section 3, we provide the existence and uniqueness result for path-dependent BSVIEs with jumps and give a stability estimate to such a solution, extending the results of Peng and Wang (2016) on path-dependent BSDE without jumps and Yong (2008). In Section 4, we study the path-differentiability of solutions to path-dependent BSVIEs with jumps, as done for BSDEs in Kromer et al. (2017). In Section 5, we will establish a duality principle between a linear path-dependent FSVIE with jumps and a linear path-dependent BSVIE with jumps as well as a comparison theorem for adapted M-solutions to BSVIEs, again generalizing the results in Wang and Yong (2015) by introducing pathdependency and jumps. Finally, a class of dynamic coherent risk measures based on path-dependent BSVIEs with jumps are derived in Section 6.

\section{Preliminaries}

Let $t \geq 0$ and $T>t$ be fixed. Throughout this paper, we consider a probability space $(\Omega, \mathbb{F}, \mathbb{P})$ with the filtration $\mathbb{F}=\left(\mathcal{F}_{s}^{t}\right)_{s \in[t, T]}$ defined by $\mathcal{F}_{s}^{t}=$ $\sigma\left\{W(r)-W(t), t \leq r \leq s ; N(I, A), I \subseteq(t, s), A \subseteq \mathbb{R}^{l} ; \mathbb{1}_{B}, B \in \mathcal{N}\right\}$, where $(W(s))_{s \in[0, T]}$ is a $d$-dimensional Brownian motion, $N$ a random measure, and $\mathcal{N}$ the $\mathbb{P}$-null set.

The space of all càdlàg $\mathbb{R}^{d}$-valued functions on $[0, T]$ endowed with the supremum metric is denoted by $D\left([0, T], \mathbb{R}^{d}\right)$. For each $\gamma \in D\left([0, T], \mathbb{R}^{d}\right)$ the value of $\gamma$ at $s \in[0, T]$ is $\gamma(s)$ and we define the path of $\gamma$ stopped at $t \in[0, T]$, denoted by $\gamma_{t}$, as $\gamma_{t}(u)=\gamma(u) \mathbb{1}_{[0, t)}(u)+\gamma(t) \mathbb{1}_{[t, T]}(u)=\gamma(u \wedge t)$ for all $u \in[0, T]$. The path shifted by $h \in \mathbb{R}^{d}$ in $t$ is denoted by $\gamma_{t}^{h}(u)=\gamma(u \wedge t)+h \mathbb{1}_{[t, T]}(u)$, for $u \in[0, T]$. Furthermore, for $t, \bar{t} \in[0, T]$ it is 
$\left\|\gamma_{t}-\bar{\gamma}_{\bar{t}}\right\|_{D}:=\left\|\gamma_{t}-\bar{\gamma}_{\bar{t}}\right\|_{D(t, \bar{t})}=\left\|\gamma_{t}-\bar{\gamma}_{\bar{t}}\right\|+|t-\bar{t}|=\sup _{s \in[0, T]}\left|\gamma_{t}(s)-\bar{\gamma}_{\bar{t}}(s)\right|+|t-\bar{t}|$.

For any $\gamma \in D\left([0, T], \mathbb{R}^{d}\right)$ and a fixed $t \in[0, T]$, we are looking for a unique solution $\left(Y_{\gamma_{t}}(\cdot), Z_{\gamma_{t}}(\cdot, \cdot), U_{\gamma_{t}}(\cdot, \cdot, \cdot)\right)$ to the following path-dependent backward stochastic Volterra integral equation (BSVIE) with jumps

$$
\begin{aligned}
Y_{\gamma_{t}}(s)= & \Phi\left(X_{\gamma_{t}, s}\right)+\int_{s}^{T} f\left(s, r, X_{\gamma_{t}, r-}, Y_{\gamma_{t}}(r-), Z_{\gamma_{t}}(s, r), Z_{\gamma_{t}}(r, s), U_{\gamma_{t}}(s, r), U_{\gamma_{t}}(r, s)\right) d r \\
& -\int_{s}^{T} Z_{\gamma_{t}}(s, r) d W(r)-\int_{s}^{T} \int_{\mathbb{R}^{l}} U_{\gamma_{t}}(s, r, z) \tilde{N}(d r, d z),
\end{aligned}
$$

where $X_{\gamma_{t}}$ is a $\mathbb{R}^{d}$-valued adapted càdlàg process on $[t, T]$ with initial path $\gamma \in$ $D\left([0, T], \mathbb{R}^{d}\right) . N(d s, d x)$ is an integer-valued random measure and $\tilde{N}(d s, d z)=$ $N(d s, d z)-v(d z) d s$ is the compensated Poisson measure with a Lévy measure $v$. For the definition of the spaces that we need for the later sections, we use the notation of Delong (2013), Levental et al. (2013), Peng and Wang (2016) and Yong (2008).

- Let $\mathcal{M}\left(\mathbb{R}^{l} ; \mathbb{R}^{m}\right)$ be the space of all measurable functions from $\mathbb{R}^{l}$ to $\mathbb{R}^{m}$.

- $\quad L_{\mu}^{2}\left(\mathbb{R}^{l} ; \mathbb{R}^{m}\right)$ denotes the space of all $\varphi \in \mathcal{M}\left(\mathbb{R}^{l} ; \mathbb{R}^{m}\right)$ which satisfy

$$
\int_{\mathbb{R}^{l}}|\varphi(x)|^{2} \mu(d x)<\infty .
$$

- Let

$$
\begin{aligned}
L_{\mathcal{F}_{T}^{t}}^{2}\left(D\left([0, T], \mathbb{R}^{d}\right) ; \mathbb{R}^{m}\right)= & \left\{\psi: \Omega \times D\left([0, T], \mathbb{R}^{d}\right) \rightarrow \mathbb{R}^{m} \mid \psi(\cdot) \text { is } \mathcal{F}_{T}^{t}\right. \text {-measurable } \\
& \text { and } \left.\mathbb{E}\left[|\psi(\cdot)|^{2}\right]<\infty\right\}
\end{aligned}
$$

- Similarly, we define

$$
L_{\mathbb{F}}^{2}(t, T)=\left\{h: \Omega \times[t, T] \rightarrow \mathbb{R}^{m} \mid h(s) \text { is } \mathcal{F}_{s}^{t} \text {-measurable, } \mathbb{E}\left[\int_{t}^{T}|h(s)|^{2} d r\right]<\infty\right\}
$$

and

$$
\begin{aligned}
& L_{\mathcal{F}_{S}^{t}}^{2}(R, T) \\
& =\left\{\psi:[R, T] \times \Omega \rightarrow \mathbb{R}^{m} \mid \psi(s) \text { is } \mathcal{F}_{S}^{t} \text {-measurable, } \mathbb{E}\left[\int_{R}^{T}|\psi(u)|^{2} d u\right]<\infty\right\} .
\end{aligned}
$$

- We denote by $S^{2}[t, T]$ the space for the process $Y$ with

$$
\begin{aligned}
S^{2}[t, T]= & \left\{Y: \Omega \times[t, T] \rightarrow \mathbb{R}^{m} \mid(Y(s))_{s \in[t, T]} \text { is a càdlàg } \mathbb{F}\right. \text {-adapted process and } \\
& \left.\mathbb{E}\left[\sup _{s \in[t, T]}|Y(s)|^{2}\right]<\infty\right\} .
\end{aligned}
$$


- For the process $Z$, we introduce the space

$$
\begin{aligned}
M^{2}[t, T]= & \left\{Z: \Omega \times[t, T] \rightarrow \mathbb{R}^{m \times d} \mid(Z(s))_{s \in[t, T]}\right. \text { is predictable and } \\
& \left.\mathbb{E}\left[\int_{t}^{T}\|Z(s)\|^{2} d s\right]<\infty\right\},
\end{aligned}
$$

where we denote $\|z\|=\sqrt{\operatorname{tr}\left(z z^{*}\right)}$ for $z \in \mathbb{R}^{m \times d}$.

- The space for the process $U$ is denoted by

$$
\begin{aligned}
L_{N}^{2}(t, T)= & \left\{U: \Omega \times[t, T] \times \mathbb{R}^{l} \rightarrow \mathbb{R}^{m} \mid(U(s, z))_{s \in[t, T], z \in \mathbb{R}^{l}}\right. \text { is predictable and } \\
& \left.\mathbb{E}\left[\int_{t}^{T} \int_{\mathbb{R}^{l}}|U(s, z)|^{2} v(d z) d s\right]<\infty\right\} .
\end{aligned}
$$

- We define the space $\mathbb{H}^{2}[t, T]:=S^{2}[t, T] \times M^{2}[t, T] \times L_{N}^{2}(t, T)$ and equip it with the norm

$$
\begin{aligned}
& \|(Y, Z, U)\|_{\mathbb{H}^{2}[t, T]} \\
& =\left(\mathbb{E}\left[\sup _{s \in[t, T]}|Y(s)|^{2}+\int_{t}^{T}\|Z(r)\|^{2} d r+\int_{t}^{T} \int_{\mathbb{R}^{l}}|U(r, z)|^{2} v(d z) d r\right]\right)^{\frac{1}{2}} .
\end{aligned}
$$

- We denote the space for the process $Z(\cdot, \cdot)$ of a solution to a BSVIE by

$$
\begin{aligned}
L^{2}\left(S, T ; M^{2}[R, T]\right)= & \left\{Z: \Omega \times[S, T] \times[R, T] \rightarrow \mathbb{R}^{m \times d} \mid \text { for almost all } u \in[S, T]\right. \\
& \left.Z(u, \cdot) \in M^{2}[R, T], \mathbb{E}\left[\int_{S}^{T} \int_{R}^{T}\|Z(u, r)\|^{2} d r d u\right]<\infty\right\} .
\end{aligned}
$$

- For the process $U(\cdot, \cdot, \cdot)$ of BSVIEs, we define the space

$$
\begin{aligned}
& L^{2}\left(S, T ; L_{N}^{2}(R, T)\right) \\
& =\left\{U: \Omega \times[S, T] \times[R, T] \times \mathbb{R}^{l} \rightarrow \mathbb{R}^{m} \mid \text { for almost all } u \in[S, T]\right. \\
& \left.\quad U(u, \cdot, \cdot) \in L_{N}^{2}(R, T), \mathbb{E}\left[\int_{S}^{T} \int_{R}^{T} \int_{\mathbb{R}^{l}}|U(u, r, z)|^{2} v(d z) d r d u\right]<\infty\right\} .
\end{aligned}
$$

- We define the space $\mathcal{H}^{2}[t, T]=S^{2}[t, T] \times L^{2}\left(t, T ; M^{2}[t, T]\right) \times L^{2}$ $\left(t, T ; L_{N}^{2}(t, T)\right)$ equipped with the norm

$$
\begin{aligned}
& \|(Y(\cdot), Z(\cdot, \cdot), U(\cdot, \cdot, \cdot))\|_{\mathcal{H}^{2}[t, T]} \\
& =\left(\mathbb { E } \left[\sup _{s \in[t, T]}|Y(s)|^{2}+\int_{t}^{T} \int_{t}^{T}\|Z(s, r)\|^{2} d r d s\right.\right. \\
& \left.\left.\quad+\int_{t}^{T} \int_{t}^{T} \int_{\mathbb{R}^{l}}|U(s, r, z)|^{2} v(d z) d r d s\right]\right)^{\frac{1}{2}} .
\end{aligned}
$$

Furthermore, we assume that the weak property of predictable representation (PR) holds. 
(PR) Any square integrable $\mathbb{F}$-martingale $M$ has the unique representation

$$
M(s)=M(t)+\int_{t}^{s} Z(r) d W(r)+\int_{t}^{s} \int_{\mathbb{R}^{l}} U(r, x) \tilde{N}(d r, d x), \quad s \in[t, T],
$$

where $(Z, U) \in M^{2}[t, T] \times L_{N}^{2}(t, T)$. For more details, see Section III.4 in Jacod and Shiryaev (2003) or Section XIII.2 in He et al. (1992).

For the reader's convenience, we introduce some necessary definitions and results. From Levental et al. (2013), we use the following definition about path-derivatives. These derivatives are consistent with the derivatives from Dupire (2009).

Definition 1 Let $F: D\left([0, T], \mathbb{R}^{d}\right) \rightarrow \mathbb{R}^{m}$ and $\left\{e_{i}\right\}_{i=1, \ldots, d}$ the canonical basis of $\mathbb{R}^{d}$. For $\gamma \in D\left([0, T], \mathbb{R}^{d}\right)$, we denote by $D_{i}^{1} F(\gamma ;[t, T]), i=1, \ldots, d$, the derivative of $F$ at $\gamma$ in the direction of the $\mathbb{R}^{d}$-valued process $\mathbb{1}_{[t, T]} e_{i}$, which means

$$
D_{i}^{1} F(\gamma ;[t, T])=\lim _{h \rightarrow 0} \frac{F\left(\gamma+h \mathbb{1}_{[t, T]} e_{i}\right)-F(\gamma)}{h} .
$$

By

$$
D_{i j}^{2} F(\gamma ;[t, T])=\lim _{h \rightarrow 0} \frac{D_{j}^{1} F\left(\gamma+h \mathbb{1}_{[t, T]} e_{i} ;[t, T]\right)-D_{j}^{1} F(\gamma ;[t, T])}{h}
$$

we denote the second-order derivative in the direction $1_{[t, T]} e_{i}$ and $1_{[t, T]} e_{j}$, for $i, j=$ $1, \ldots, d$. Note that the derivative $D_{i}^{1} F(\gamma ;[t, T])$ is in $\mathbb{R}^{m}$ and $D^{1} F(\gamma ;[t, T]) \in$ $\mathbb{R}^{m \times d}$. Furthermore, the second-order derivative $D_{i j}^{2} F(\gamma ;[t, T])$ is in $\mathbb{R}^{m}$ and $D^{2} F(\gamma ;[t, T])$ is a tensor. $F$ is said to be of class $C^{1}\left(D\left([0, T], \mathbb{R}^{d}\right)\right)$ if the derivatives $D_{i}^{1} F(\gamma ;[t, T])$ exist and are continuous. The class $C^{2}\left(D\left([0, T], \mathbb{R}^{d}\right)\right)$ can be defined similarly.

In order to prove our main result on BSVIE in Section 3, we introduce an auxiliary path-dependent BSDE similar to that in Wang and Yong (2015) where the simpler case without jumps and no path-dependency is considered.

First, for a given path $\gamma \in D\left([0, T], \mathbb{R}^{d}\right)$ and $t \in[0, T]$ we consider the following path-dependent BSDE with jumps

$$
\begin{aligned}
Y_{\gamma_{t}}(s)= & \Phi\left(X_{\gamma_{t}, T}\right)+\int_{s}^{T} f\left(r, X_{\gamma_{t}, r-}, Y_{\gamma_{t}}(r-), Z_{\gamma_{t}}(r), U_{\gamma_{t}}(r)\right) d r-\int_{s}^{T} Z_{\gamma_{t}}(r) d W(r) \\
& -\int_{s}^{T} \int_{\mathbb{R}^{l}} U_{\gamma_{t}}(r, z) \tilde{N}(d r, d z), \quad s \in[t, T] .
\end{aligned}
$$

We assume that

$$
\Phi \in L_{\mathcal{F}_{T}^{t}}^{2}\left(D\left([0, T], \mathbb{R}^{d}\right) ; \mathbb{R}^{m}\right)
$$


(A2) the generator $f: \Omega \times[t, T] \times D\left([0, T], \mathbb{R}^{d}\right) \times \mathbb{R}^{m} \times \mathbb{R}^{m \times d} \times \mathcal{M}\left(\mathbb{R}^{l} ; \mathbb{R}^{m}\right) \rightarrow$ $\mathbb{R}^{m}$ is $\mathbb{F}$-progressively measurable and Lipschitz continuous in the sense that

$$
\begin{aligned}
& |f(\omega, s, \gamma, y, z, u)-f(\omega, s, \bar{\gamma}, \bar{y}, \bar{z}, \bar{u})| \\
& \leq K(s)\left(\|\gamma-\bar{\gamma}\|_{D}+|y-\bar{y}|+\|z-\bar{z}\|+\int_{\mathbb{R}^{l}}|u(z)-\bar{u}(z)| v(d z)\right),
\end{aligned}
$$

a.e. $(\omega, s) \in \Omega \times[t, T]$ for all $(\gamma, y, z, u),(\bar{\gamma}, \bar{y}, \bar{z}, \bar{u}) \in D\left([0, T], \mathbb{R}^{d}\right) \times \mathbb{R}^{m} \times$ $\mathbb{R}^{m \times d} \times L_{\nu}^{2}\left(\mathbb{R}^{l} ; \mathbb{R}^{m}\right)$ and with $K:=\sup _{s \in[t, T]}|K(s)|^{2}<\infty$,

(A3) $\mathbb{E}\left[\int_{t}^{T}\left|f_{0}\left(r, \gamma_{r-}\right)\right|^{2} d r\right]<\infty$, where $f_{0}(r, \gamma):=f(r, \gamma, 0,0,0)$.

The following results can be found in Wang (2015) and Kromer et al. (2017). Since we do not need a comparison result for path-dependent BSDEs with jumps, it is sufficient to assume that the generator is progressively measurable and not predictable.

Theorem 1 Assume that (A1)-(A3) hold. For each $\gamma \in D\left([0, T], \mathbb{R}^{d}\right), t \in[0, T]$, BSDE (2) admits a unique adapted solution $\left(Y_{\gamma_{t}}, Z_{\gamma_{t}}, U_{\gamma_{t}}\right) \in \mathbb{H}^{2}[t, T]$ with

$$
Y_{\gamma_{t}}(s)=\mathbb{E}\left[\Phi\left(X_{\gamma_{t}, T}\right)+\int_{s}^{T} f\left(r, X_{\gamma_{t}, r-}, Y_{\gamma_{t}}(r-), Z_{\gamma_{t}}(r), U_{\gamma_{t}}(r)\right) d r \mid \mathcal{F}_{s}^{t}\right]
$$

and the following stability estimate holds

$$
\left\|\left(Y_{\gamma_{t}}, Z_{\gamma_{t}}, U_{\gamma_{t}}\right)\right\|_{\mathbb{H}^{2}[t, T]}^{2} \leq C\left(\mathbb{E}\left[\left|\Phi\left(X_{\gamma_{t}, T}\right)\right|^{2}\right]+\mathbb{E}\left[\int_{t}^{T}\left|f_{0}\left(r, X_{\gamma_{t}, r-}\right)\right|^{2} d r\right]\right) .
$$

Let $(\bar{f}, \bar{\Phi})$ also satisfy (A1)-(A3) and for any $\bar{\gamma} \in D\left([0, T], \mathbb{R}^{d}\right)$ let $\left(\bar{Y}_{\bar{\gamma}_{t}}, \bar{Z}_{\bar{\gamma}_{t}}, \bar{U}_{\bar{\gamma}_{t}}\right) \in \mathbb{H}^{2}[t, T]$ be the unique adapted solution to BSDE (2) with respect to $(\bar{f}, \bar{\Phi})$. Then,

$$
\begin{aligned}
& \left\|\left(Y_{\gamma_{t}}, Z_{\gamma_{t}}, U_{\gamma_{t}}\right)-\left(\bar{Y}_{\bar{\gamma}_{t}}, \bar{Z}_{\bar{\gamma}_{t}}, \bar{U}_{\bar{\gamma}_{t}}\right)\right\|_{\mathbb{H}^{2}[t, T]}^{2} \leq C \mathbb{E}\left[\left|\Phi\left(X_{\gamma_{t}, T}\right)-\bar{\Phi}\left(X_{\bar{\gamma}_{t}, T}\right)\right|^{2}\right. \\
& \left.+\int_{t}^{T}\left|f\left(r, X_{\gamma_{t}, r-}, Y_{\gamma_{t}}(r-), Z_{\gamma_{t}}(r), U_{\gamma_{t}}(r)\right)-\bar{f}\left(r, X_{\bar{\gamma}_{t}, r-}, Y_{\gamma_{t}}(r-), Z_{\gamma_{t}}(r), U_{\gamma_{t}}(r)\right)\right|^{2} d r\right] .
\end{aligned}
$$

\section{Well-posedness of path-dependent BSVIEs with jumps}

In this section, we look for a unique adapted solution to the path-dependent BSVIE (1). The notation in this section will follow the notation in Yong (2008) and Wang and Yong (2015).

For $t \leq R<S \leq T$ we denote

$$
\begin{aligned}
& \Delta[R, S]=\left\{(r, s) \in[R, S]^{2} \mid R \leq s<r \leq S\right\}, \\
& \Delta^{c}[R, S]=\left\{(r, s) \in[R, S]^{2} \mid R \leq r \leq s \leq S\right\} .
\end{aligned}
$$


In contrast to path-dependent BSDEs with jumps, the generator of a BSVIE depends on both $Z(s, r), U(s, r)$ and $Z(r, s), U(r, s)$. If the processes $\left(Y_{\gamma_{t}}(\cdot), Z_{\gamma_{t}}(\cdot, \cdot), U_{\gamma_{t}}(\cdot, \cdot, \cdot)\right)$ satisfy a path-dependent BSVIE with jumps in the usual Itô sense, the processes $Z_{\gamma_{t}}$ and $U_{\gamma_{t}}$ of the solution are only unique determined on $\Delta^{c}[t, T]$, as shown in Example 1.1. in Yong (2008). There a more restrictive notion of solution is also introduced which we modify for our purpose.

Definition 2 Let $t \in[0, T], S \in[t, T)$ and $\gamma \in D\left([0, T], \mathbb{R}^{d}\right)$. $\left(Y_{\gamma_{t}}(\cdot), Z_{\gamma_{t}}(\cdot, \cdot), U_{\gamma_{t}}(\cdot, \cdot, \cdot)\right) \in \mathcal{H}^{2}[S, T]$ is called an adapted $M$-solution to BSVIE (1) on $[S, T]$ if (1) holds in the usual Itô sense for almost all $s \in[S, T]$ and, in addition, the following holds

$$
\begin{aligned}
& Y_{\gamma_{t}}(u) \\
& =\mathbb{E}\left[Y_{\gamma_{t}}(u) \mid \mathcal{F}_{S}^{t}\right]+\int_{S}^{u} Z_{\gamma_{t}}(u, r) d W(r)+\int_{S}^{u} \int_{\mathbb{R}^{l}} U_{\gamma_{t}}(u, r, z) \tilde{N}(d r, d z), \text { a.e. } u \in[S, T] .
\end{aligned}
$$

Remark 1 If $\left(Y_{\gamma_{t}}(\cdot), Z_{\gamma_{t}}(\cdot, \cdot), U_{\gamma_{t}}(\cdot, \cdot, \cdot)\right) \in \mathcal{H}^{2}[S, T]$ is an adapted M-solution to BSVIE (1) on $[S, T]$, it is also an adapted M-solution on $[\bar{S}, T]$ for any $\bar{S} \in(S, T)$.

For any $\bar{S} \in(S, T)$ and almost all $u \in[\bar{S}, T]$, with Eq. (6) is

$$
\mathbb{E}\left[Y_{\gamma_{t}}(u) \mid \mathcal{F}_{\bar{S}}^{t}\right]=\mathbb{E}\left[Y_{\gamma_{t}}(u) \mid \mathcal{F}_{S}^{t}\right]+\int_{S}^{\bar{S}} Z_{\gamma_{t}}(u, r) d W(r)+\int_{S}^{\bar{S}} \int_{\mathbb{R}^{l}} U_{\gamma_{t}}(u, r, z) \tilde{N}(d r, d z) .
$$

This yields for almost all $u \in[\bar{S}, T]$

$$
\begin{aligned}
Y_{\gamma_{t}}(u)= & \mathbb{E}\left[Y_{\gamma_{t}}(u) \mid \mathcal{F}_{\bar{S}}^{t}\right]-\int_{S}^{\bar{S}} Z_{\gamma_{t}}(u, r) d W(r)-\int_{S}^{\bar{S}} \int_{\mathbb{R}^{l}} U_{\gamma_{t}}(u, r, z) \tilde{N}(d r, d z) \\
& +\int_{S}^{u} Z_{\gamma_{t}}(u, r) d W(r)+\int_{S}^{u} \int_{\mathbb{R}^{l}} U_{\gamma_{t}}(u, r, z) \tilde{N}(d r, d z) \\
= & \mathbb{E}\left[Y_{\gamma_{t}}(u) \mid \mathcal{F}_{\bar{S}}^{t}\right]+\int_{\bar{S}}^{u} Z_{\gamma_{t}}(u, r) d W(r)+\int_{\bar{S}}^{u} \int_{\mathbb{R}^{l}} U_{\gamma_{t}}(u, r, z) \tilde{N}(d r, d z) .
\end{aligned}
$$

As in Yong (2008), we prove existence and uniqueness of a solution to the pathdependent BSVIE with jumps (1) where the generator is independent of $Y_{\gamma_{t}}(u)$, $Z_{\gamma_{t}}(u, s)$ and $U_{\gamma_{t}}(u, s, z)$ for $(s, u, z) \in \Delta[t, T] \times \mathbb{R}^{l}$. For the proof we use an auxiliary stochastic integral equation of the form

$$
\begin{aligned}
\lambda_{\gamma_{t}}(s, r)= & \Phi\left(X_{\gamma_{t}, s}\right)+\int_{r}^{T} h\left(s, u, X_{\gamma_{t}, u-}, \mu_{\gamma_{t}}(s, u), \varphi_{\gamma_{t}}(s, u)\right) d u-\int_{r}^{T} \mu_{\gamma_{t}}(s, u) d W(u) \\
& -\int_{r}^{T} \int_{\mathbb{R}^{l}} \varphi_{\gamma_{t}}(s, u, z) \tilde{N}(d u, d z), \quad r \in[R, T], s \in[S, T],
\end{aligned}
$$

where $R, S \in[t, T)$ are given, the process $\lambda_{\gamma_{t}}(s, \cdot)$ is $\mathbb{F}$-adapted, and $\mu_{\gamma_{t}}(s, \cdot)$, $\varphi_{\gamma_{t}}(s, \cdot, \cdot)$ are predictable for all $s \in[S, T]$. For any $s \in[S, T]$ this stochastic integral equation (6) is a path-dependent BSDE with jumps on $[R, T]$. Similar to Yong (2008), we assume 
(B1) $\Phi \in L_{\mathcal{F}_{T}^{t}}^{2}\left(D\left([0, T], \mathbb{R}^{d}\right) ; \mathbb{R}^{m}\right)$.

$$
h: \Omega \times[S, T] \times[R, T] \times D\left([0, T], \mathbb{R}^{d}\right) \times \mathbb{R}^{m \times d} \times \mathcal{M}\left(\mathbb{R}^{l} ; \mathbb{R}^{m}\right) \rightarrow \mathbb{R}^{m}
$$

is $\mathcal{F}_{T}^{t} \otimes \mathcal{B}\left([S, T] \times[R, T] \times D\left([0, T], \mathbb{R}^{d}\right) \times \mathbb{R}^{m \times d} \times \mathcal{M}\left(\mathbb{R}^{l} ; \mathbb{R}^{m}\right)\right)$-measurable such that $r \mapsto h(s, r, \gamma, z, u)$ is $\mathbb{F}$-progressive measurable for all $(s, \gamma, z, u) \in$ $[S, T] \times D\left([0, T], \mathbb{R}^{d}\right) \times \mathbb{R}^{m \times d} \times L_{v}^{2}\left(\mathbb{R}^{l}, \mathbb{R}^{m}\right)$. Furthermore, the generator $h$ is Lipschitz continuous in the sense that

$$
\begin{aligned}
& |h(\omega, s, r, \gamma, z, u)-h(\omega, s, r, \bar{\gamma}, \bar{z}, \bar{u})| \\
& \leq K(s, r)\left(\|\gamma-\bar{\gamma}\|_{D}+\|z-\bar{z}\|+\int_{\mathbb{R}^{l}}|u(x)-\bar{u}(x)| v(d x)\right),
\end{aligned}
$$

a.e. $(\omega, s, r) \in \Omega \times[S, T] \times[R, T]$ for all $(\gamma, z, u) \in D\left([0, T], \mathbb{R}^{d}\right) \times \mathbb{R}^{m \times d} \times$ $L_{v}^{2}\left(\mathbb{R}^{l}, \mathbb{R}^{m}\right) . K:[S, T] \times[R, T] \rightarrow[0, \infty)$ is a deterministic function such that for $\varepsilon>0$,

$$
\sup _{s \in[S, T]} \int_{R}^{T}|K(s, r)|^{2+\varepsilon} d r<\infty .
$$

(B3) $\mathbb{E}\left[\int_{S}^{T} \int_{R}^{T}\left|h_{0}\left(s, u, \gamma_{u-}\right)\right|^{2} d u d s\right]<\infty$, where $h_{0}(s, u, \gamma):=h(s, u$, $\gamma, 0,0)$.

In the special case for $R=S$ with given $S \in[t, T)$, we define

$$
\begin{aligned}
Y_{\gamma_{t}}(s) & =\lambda_{\gamma_{t}}(s, s), \quad s \in[S, T], \\
Z_{\gamma_{t}}(s, u) & =\mu_{\gamma_{t}}(s, u), \quad(s, u) \in \Delta^{c}[S, T], \\
U_{\gamma_{t}}(s, u, z) & =\varphi_{\gamma_{t}}(s, u, z), \quad(s, u) \in \Delta^{c}[S, T], z \in \mathbb{R}^{l} .
\end{aligned}
$$

Similar to Yong (2008), we get for this special case of the auxiliary stochastic integral Eq. (7) the following result.

Corollary 1 Let (B1)-(B3) hold. For any $\gamma \in D\left([0, T], \mathbb{R}^{d}\right)$ and $t \in[0, T] a$ path-dependent BSVIE with jumps of the form

$$
\begin{aligned}
Y_{\gamma_{t}}(s)= & \Phi\left(X_{\gamma_{t}, s}\right)+\int_{s}^{T} h\left(s, u, X_{\gamma_{t}, u-}, Z_{\gamma_{t}}(s, u), U_{\gamma_{t}}(s, u)\right) d u-\int_{s}^{T} Z_{\gamma_{t}}(s, u) d W(u) \\
& -\int_{s}^{T} \int_{\mathbb{R}^{l}} U_{\gamma_{t}}(s, u, z) \tilde{N}(d u, d z), \quad s \in[S, T],
\end{aligned}
$$

admits a unique adapted M-solution $\left(Y_{\gamma_{t}}(\cdot), Z_{\gamma_{t}}(\cdot, \cdot), U_{\gamma_{t}}(\cdot, \cdot, \cdot)\right) \in \mathcal{H}^{2}[S, T]$ and the following estimates hold

$$
\begin{aligned}
& \left\|\left(Y_{\gamma_{t}}(\cdot), Z_{\gamma_{t}}(\cdot, \cdot), U_{\gamma_{t}}(\cdot, \cdot, \cdot)\right)\right\|_{\mathcal{H}^{2}[S, T]}^{2} \\
& \leq C\left(\mathbb{E}\left[\int_{S}^{T}\left|\Phi\left(X_{\gamma_{t}, s-}\right)\right|^{2} d s\right]+\mathbb{E}\left[\int_{S}^{T} \int_{S}^{T}\left|h_{0}\left(s, u, X_{\gamma_{t}, u-}\right)\right|^{2} d u d s\right]\right)
\end{aligned}
$$


and for $T-S$ small

$$
\begin{aligned}
& \left\|\left(Y_{\gamma_{t}}(\cdot), Z_{\gamma_{t}}(\cdot, \cdot), U_{\gamma_{t}}(\cdot, \cdot, \cdot)\right)\right\|_{\mathcal{H}^{2}[S, T]}^{2} \\
& \leq C\left(\mathbb{E}\left[\int_{S}^{T}\left|\Phi\left(X_{\gamma_{t}, s-}\right)\right|^{2} d s\right]+\mathbb{E}\left[\int_{S}^{T}\left(\int_{S}^{T}\left|h_{0}\left(s, u, X_{\gamma_{t}, u-}\right)\right| d u\right)^{2} d s\right]\right) .
\end{aligned}
$$

Let $\bar{h}$ and $\bar{\Phi}$ also satisfy assumptions (B1)-(B3) and for a given path $\bar{\gamma} \in$ $D\left([0, T], \mathbb{R}^{d}\right)$ let $\left(Y_{\gamma_{t}}(\cdot), Z_{\gamma_{t}}(\cdot, \cdot), U_{\gamma_{t}}(\cdot, \cdot, \cdot)\right)$ be the unique adapted M-solution to BSVIE (8) with generator $\bar{h}$ and terminal condition $\bar{\Phi}$. For $T-S$ small enough, we get the estimate

$$
\begin{aligned}
& \mathbb{E}\left[\sup _{s \in[S, T]}\left|Y_{\gamma_{t}}(s)-\bar{Y}_{\bar{\gamma}_{t}}(s)\right|^{2}+\int_{S}^{T}\left\|Z_{\gamma_{t}}(s, r)-\bar{Z}_{\bar{\gamma}_{t}}(s, r)\right\|^{2} d r\right. \\
&\left.\quad+\int_{S}^{T} \int_{\mathbb{R}^{l}}\left|U_{\gamma_{t}}(s, r, z)-\bar{U}_{\bar{\gamma}_{t}}(s, r, z)\right|^{2} v(d z) d r\right] \\
& \leq C\left(\mathbb{E}\left[\left|\Phi\left(X_{\gamma_{t}, s}\right)-\bar{\Phi}\left(X_{\bar{\gamma}_{t}, s}\right)\right|^{2}\right]+\mathbb{E}\left[\left(\int_{S}^{T} \mid h\left(s, u, X_{\gamma_{t}, u-}, Z_{\gamma_{t}}(s, u), U_{\gamma_{t}}(s, u)\right)\right.\right.\right. \\
&\left.\left.\left.-\bar{h}\left(s, u, X_{\bar{\gamma}_{t}, u-}, Z_{\gamma_{t}}(s, u), U_{\gamma_{t}}(s, u)\right) \mid d u\right)^{2}\right]\right), \quad \text { a.e. } s \in[S, T] .
\end{aligned}
$$

Now, we show existence and uniqueness of a solution to a path-dependent BSVIE with jumps where the generator depends on processes $Y_{\gamma_{t}}(s), Z_{\gamma_{t}}(u, s)$ and $U_{\gamma_{t}}(u, s, z)$ for $(s, u) \in \Delta[t, T] \times \mathbb{R}^{l}$. The following assumptions are needed.

(H1) $\Phi \in L_{\mathcal{F}_{T}^{t}}^{2}\left(D\left([0, T], \mathbb{R}^{d}\right) ; \mathbb{R}^{m}\right)$.

(H2) The generator $f: \Omega \times \Delta^{c}[t, T] \times D\left([0, T], \mathbb{R}^{d}\right) \times \mathbb{R}^{m} \times \mathbb{R}^{m \times d} \times \mathbb{R}^{m \times d} \times$ $\mathcal{M}\left(\mathbb{R}^{l} ; \mathbb{R}^{m}\right) \times \mathcal{M}\left(\mathbb{R}^{l} ; \mathbb{R}^{m}\right) \rightarrow \mathbb{R}^{m}$ is $\mathcal{F}_{T}^{t} \otimes \mathcal{B}\left(\Delta^{c}[t, T] \times D\left([0, T], \mathbb{R}^{d}\right) \times\right.$ $\left.\mathbb{R}^{m} \times \mathbb{R}^{m \times d} \times \mathbb{R}^{m \times d} \times \mathcal{M}\left(\mathbb{R}^{l} ; \mathbb{R}^{m}\right) \times \mathcal{M}\left(\mathbb{R}^{l} ; \mathbb{R}^{m}\right)\right)$-measurable such that $r \mapsto$ $f(s, r, \gamma, y, z, \zeta, u, \xi)$ is $\mathbb{F}$-progressive measurable. Furthermore, generator $f$ is Lipschitz continuous in the sense that

$$
\begin{aligned}
& |f(\omega, s, r, \gamma, y, z, \zeta, u, \xi)-f(\omega, s, r, \bar{\gamma}, \bar{y}, \bar{z}, \bar{\zeta}, \bar{u}, \bar{\xi})| \\
& \leq K(s, r)\left(\|\gamma-\bar{\gamma}\|_{D}+|y-\bar{y}|+\|z-\bar{z}\|+\|\zeta-\bar{\zeta}\|+\int_{\mathbb{R}^{l}}|u(x)-\bar{u}(x)| v(d x)\right. \\
& \left.\quad+\int_{\mathbb{R}^{l}}|\xi(x)-\bar{\xi}(x)| v(d x)\right),
\end{aligned}
$$

a.e. $(\omega, s, r) \in \Omega \times \Delta^{c}[t, T]$ for all $(\gamma, y, z, \zeta, u, \xi),(\bar{\gamma}, \bar{y}, \bar{z}, \bar{\zeta}, \bar{u}, \bar{\xi}) \in$ $D\left([0, T], \mathbb{R}^{d}\right) \times \mathbb{R}^{m} \times \mathbb{R}^{m \times d} \times \mathbb{R}^{m \times d} \times L_{v}^{2}\left(\mathbb{R}^{l} ; \mathbb{R}^{m}\right) \times L_{v}^{2}\left(\mathbb{R}^{l} ; \mathbb{R}^{m}\right)$. Moreover, $K: \Delta^{c}[t, T] \rightarrow[0, \infty)$ is a deterministic function such that for $\varepsilon>0$,

$$
\sup _{s \in[t, T]} \int_{s}^{T}|K(s, r)|^{2+\varepsilon} d r<\infty .
$$


(H3) $\mathbb{E}\left[\int_{t}^{T} \int_{t}^{T}\left|f_{0}\left(s, r, \gamma_{r-}\right)\right|^{2} d r d s\right]<\infty$, where $f_{0}\left(s, r, \gamma_{r-}\right)=f\left(s, r, \gamma_{r_{-}}\right.$, $0,0,0,0,0)$.

Theorem 2 Let (H1)-(H3) hold. Then, for any given path $\gamma \in D\left([0, T], \mathbb{R}^{d}\right), t \in$ $[0, T]$, BSVIE (1) admits a unique adapted M-solution $\left(Y_{\gamma_{t}}(\cdot), Z_{\gamma_{t}}(\cdot, \cdot), U_{\gamma_{t}}(\cdot, \cdot, \cdot)\right) \in$ $\mathcal{H}^{2}[t, T]$. Moreover, the following estimate holds for all $S \in[t, T]$

$$
\begin{aligned}
& \left\|\left(Y_{\gamma_{t}}(\cdot), Z_{\gamma_{t}}(\cdot, \cdot), U_{\gamma_{t}}(\cdot, \cdot, \cdot)\right)\right\|_{\mathcal{H}^{2}[S, T]}^{2} \\
& \leq C\left(\mathbb{E}\left[\int_{S}^{T}\left|\Phi\left(X_{\gamma_{t}, s-}\right)\right|^{2} d s\right]+\mathbb{E}\left[\int_{S}^{T} \int_{S}^{T}\left|f_{0}\left(s, r, X_{\gamma_{t}, r-}\right)\right|^{2} d r d s\right]\right) .
\end{aligned}
$$

Proof We use the techniques of Yong (2008) and split this proof into four steps.

First step: We show the existence and uniqueness of the adapted M-solution to BSVIE (1) on $[S, T]$ for some $S \in[t, T)$ and given $\gamma \in D\left([0, T], \mathbb{R}^{d}\right), t \in[0, T]$. For any $S \in[t, T)$ we define the space

$$
\begin{aligned}
\mathbb{M}^{2}[S, T]= & \left\{(y(\cdot), z(\cdot, \cdot), u(\cdot, \cdot, \cdot)) \in \mathcal{H}^{2}[S, T] \mid y(s)=\mathbb{E}\left[y(s) \mid \mathcal{F}_{S}^{t}\right]\right. \\
& \left.+\int_{S}^{s} z(s, r) d W(r)+\int_{S}^{s} \int_{\mathbb{R}^{l}} u(s, r, z) \tilde{N}(d r, d z), s \in[S, T]\right\} .
\end{aligned}
$$

For $(y(\cdot), z(\cdot, \cdot), u(\cdot, \cdot, \cdot)) \in \mathbb{M}^{2}[S, T]$ we get

$$
\begin{aligned}
& \mathbb{E}\left[\sup _{s \in[S, T]}|y(s)|^{2}+\int_{S}^{T} \int_{s}^{T}\|z(s, r)\|^{2} d r d s+\int_{S}^{T} \int_{s}^{T} \int_{\mathbb{R}^{l}}|u(s, r, z)|^{2} v(d z) d r d s\right] \\
& \leq \mathbb{E}\left[\sup _{s \in[S, T]}|y(s)|^{2}+\int_{S}^{T} \int_{s}^{T}\|z(s, r)\|^{2} d r d s+\int_{S}^{T} \int_{s}^{T} \int_{\mathbb{R}^{l}}|u(s, r, z)|^{2} v(d z) d r d s\right] \\
& +\mathbb{E}\left[\int_{S}^{T} \int_{S}^{s}\|z(s, r)\|^{2} d r d s+\int_{S}^{T} \int_{S}^{s} \int_{\mathbb{R}^{l}}|u(s, r, z)|^{2} v(d z) d r d s\right] \\
& \leq C \mathbb{E}\left[\sup _{s \in[S, T]}|y(s)|^{2}+\int_{S}^{T} \int_{S}^{T}\|z(s, r)\|^{2} d r d s+\int_{S}^{T} \int_{S}^{T} \int_{\mathbb{R}^{l}}|u(s, r, z)|^{2} v(d z) d r d s\right] .
\end{aligned}
$$

Thus,

$$
\begin{aligned}
& \|(y(\cdot), z(\cdot, \cdot), u(\cdot, \cdot, \cdot))\|_{\mathbb{M}^{2}[S, T]}^{2} \\
& =\mathbb{E}\left[\sup _{s \in[S, T]}|y(s)|^{2}+\int_{S}^{T} \int_{S}^{T}\|z(s, r)\|^{2} d r d s+\int_{S}^{T} \int_{S}^{T} \int_{\mathbb{R}^{l}}|u(s, r, z)|^{2} v(d z) d r d s\right]
\end{aligned}
$$


is an equivalent norm for $\mathbb{M}^{2}[S, T]$. Now, we consider the following path-dependent BSVIE with jumps

$$
\begin{aligned}
Y_{\gamma_{t}}(s)= & \Phi\left(X_{\gamma_{t}, s}\right)+\int_{s}^{T} f\left(s, r, X_{\gamma_{t}, r-}, y_{\gamma_{t}}(r-), Z_{\gamma_{t}}(s, r), z_{\gamma_{t}}(r, s), U_{\gamma_{t}}(s, r), u_{\gamma_{t}}(r, s)\right) d r \\
& -\int_{s}^{T} Z_{\gamma_{t}}(s, r) d W(r)-\int_{s}^{T} \int_{\mathbb{R}^{l}} U_{\gamma_{t}}(s, r, z) \tilde{N}(d r, d z), \quad s \in[S, T],
\end{aligned}
$$

where $\left(y_{\gamma_{t}}(\cdot), z_{\gamma_{t}}(\cdot, \cdot), u_{\gamma_{t}}(\cdot, \cdot, \cdot)\right) \in \mathbb{M}^{2}[S, T]$ are given. Corollary 1 yields that this BSVIE admits a unique adapted M-solution $\left(Y_{\gamma_{t}}(\cdot), Z_{\gamma_{t}}(\cdot, \cdot), U_{\gamma_{t}}(\cdot, \cdot, \cdot)\right) \in$ $\mathcal{H}^{2}[S, T]$. With Eq. (10) the following estimate holds for $T-S$ small

$$
\begin{aligned}
& \mathbb{E}\left[\sup _{s \in[S, T]}\left|Y_{\gamma_{t}}(s)\right|^{2}+\int_{s}^{T}\left\|Z_{\gamma_{t}}(s, r)\right\|^{2} d r+\int_{s}^{T} \int_{\mathbb{R}^{l}}\left|U_{\gamma_{t}}(s, r, z)\right|^{2} v(d z) d r\right] \\
& \leq C\left(\mathbb{E}\left[\left|\Phi\left(X_{\gamma_{t}, s}\right)\right|^{2}\right]+2 \mathbb{E}\left[\left(\int_{S}^{T}\left|f_{0}\left(s, r, X_{\gamma_{t}, r-}\right)\right| d r\right)^{2}\right]\right. \\
& \left.+2 \mathbb{E}\left[\left(\int_{S}^{T} K(s, r)\left(\left|y_{\gamma_{t}}(r-)\right|+\left\|z_{\gamma_{t}}(r, s)\right\|+\int_{\mathbb{R}^{l}}\left|u_{\gamma_{t}}(r, s, z)\right| v(d z)\right) d r\right)^{2}\right]\right),
\end{aligned}
$$

a.e. $s \in[S, T]$.

Hölder's inequality yields

$$
\begin{aligned}
& \mathbb{E}\left[\sup _{s \in[S, T]}\left|Y_{\gamma_{t}}(s)\right|^{2}+\int_{s}^{T}\left\|Z_{\gamma_{t}}(s, r)\right\|^{2} d r+\int_{s}^{T} \int_{\mathbb{R}^{l}}\left|U_{\gamma_{t}}(s, r, z)\right|^{2} v(d z) d r\right] \\
& \leq 2 C\left(\mathbb{E}\left[\left|\Phi\left(X_{\gamma_{t}, s}\right)\right|^{2}\right]+\mathbb{E}\left[\left(\int_{S}^{T}\left|f_{0}\left(s, r, X_{\gamma_{t}, r-}\right)\right| d r\right)^{2}\right]+\mathbb{E}\left[\left(\int_{S}^{T}|K(s, r)|^{2+\varepsilon} d r\right)^{\frac{2}{2+\varepsilon}}\right.\right. \\
& \left.\left.\cdot(T-S)^{\frac{\varepsilon}{2+\varepsilon}}\left(\sup _{s \in[S, T]}\left|y_{\gamma_{t}}(s)\right|^{2}+\int_{S}^{T}\left\|z_{\gamma_{t}}(r, s)\right\|^{2} d r+\int_{S}^{T} \int_{\mathbb{R}^{l}}\left|u_{\gamma_{t}}(r, s, z)\right|^{2} v(d z) d r\right)\right]\right) .
\end{aligned}
$$

It is

$$
\begin{aligned}
& \mathbb{E}\left[\sup _{s \in[S, T]}\left|y_{\gamma_{t}}(s)\right|^{2}+\int_{S}^{T} \int_{S}^{T}\left\|z_{\gamma_{t}}(r, s)\right\|^{2} d r d s+\int_{S}^{T} \int_{S}^{T} \int_{\mathbb{R}^{l}}\left|u_{\gamma_{t}}(r, s, z)\right|^{2} v(d z) d r d s\right] \\
& \leq \mathbb{E}\left[\sup _{s \in[S, T]}\left|y_{\gamma_{t}}(s)\right|^{2}+\int_{S}^{T} \int_{S}^{T}\left\|z_{\gamma_{t}}(r, s)\right\|^{2} d s d r+\int_{S}^{T} \int_{S}^{T} \int_{\mathbb{R}^{l}}\left|u_{\gamma_{t}}(r, s, z)\right|^{2} v(d z) d s d r\right] \\
& \leq C \mathbb{E}\left[\sup _{s \in[S, T]}\left|y_{\gamma_{t}}(s)\right|^{2}+\int_{S}^{T} \int_{r}^{T}\left\|z_{\gamma_{t}}(r, s)\right\|^{2} d s d r\right. \\
& \left.\quad+\int_{S}^{T} \int_{r}^{T} \int_{\mathbb{R}^{l}}\left|u_{\gamma_{t}}(r, s, z)\right|^{2} v(d z) d s d r\right]
\end{aligned}
$$


and this implies

$$
\begin{aligned}
& \left\|\left(Y_{\gamma_{t}}(\cdot), Z_{\gamma_{t}}(\cdot, \cdot), U_{\gamma_{t}}(\cdot, \cdot, \cdot)\right)\right\|_{\mathbb{M}^{2}[S, T]}^{2} \\
& \leq C\left\{\mathbb{E}\left[\int_{S}^{T}\left|\Phi\left(X_{\gamma_{t}, s-}\right)\right|^{2} d s\right]+\mathbb{E}\left[\int_{S}^{T} \int_{S}^{T}\left|f_{0}\left(s, r, X_{\gamma_{t}, r-}\right)\right|^{2} d r d s\right]\right. \\
& \left.\quad+\left\|\left(y_{\gamma_{t}}(\cdot), z_{\gamma_{t}}(\cdot, \cdot), u_{\gamma_{t}}(\cdot, \cdot, \cdot)\right)\right\|_{\mathbb{M}^{2}[S, T]}^{2}\right\} .
\end{aligned}
$$

Therefore, $\left(Y_{\gamma_{t}}(\cdot), Z_{\gamma_{t}}(\cdot, \cdot), U_{\gamma_{t}}(\cdot, \cdot, \cdot)\right)$ is in $\mathbb{M}^{2}[S, T]$. Now, we define a map $\Theta^{t, \gamma}: \mathbb{M}^{2}[S, T] \rightarrow \mathbb{M}^{2}[S, T]$ by

$$
\Theta^{t, \gamma}\left(y_{\gamma_{t}}(\cdot), z_{\gamma_{t}}(\cdot, \cdot), u_{\gamma_{t}}(\cdot, \cdot, \cdot)\right)=\left(Y_{\gamma_{t}}(\cdot), Z_{\gamma_{t}}(\cdot, \cdot), U_{\gamma_{t}}(\cdot, \cdot, \cdot)\right)
$$

for all $\left(y_{\gamma_{t}}(\cdot), z_{\gamma_{t}}(\cdot, \cdot), u_{\gamma_{t}}(\cdot, \cdot, \cdot)\right) \in \mathbb{M}^{2}[S, T]$. Furthermore, let $\Theta^{t, \gamma}\left(\bar{y}_{\gamma_{t}}(\cdot)\right.$, $\left.\bar{z}_{\gamma_{t}}(\cdot),, \bar{u}_{\gamma_{t}}(\cdot, \cdot, \cdot)\right)=\left(\bar{Y}_{\gamma_{t}}(\cdot), \bar{Z}_{\gamma_{t}}(\cdot, \cdot), \bar{U}_{\gamma_{t}}(\cdot, \cdot, \cdot)\right)$ for $\left(\bar{y}_{\gamma_{t}}(\cdot), \bar{z}_{\gamma_{t}}(\cdot, \cdot), \bar{u}_{\gamma_{t}}(\cdot, \cdot, \cdot)\right) \in$ $\mathbb{M}^{2}[S, T]$. Corollary 1 yields for $T-S$ small

$$
\begin{aligned}
& \mathbb{E}\left[\sup _{s \in[S, T]}\left|Y_{\gamma_{t}}(s)-\bar{Y}_{\gamma_{t}}(s)\right|^{2}+\int_{s}^{T}\left\|Z_{\gamma_{t}}(s, r)-\bar{Z}_{\gamma_{t}}(s, r)\right\|^{2} d r\right. \\
& \left.+\int_{s}^{T} \int_{\mathbb{R}^{l}}\left|U_{\gamma_{t}}(s, r, z)-\bar{U}_{\gamma_{t}}(s, r, z)\right|^{2} v(d z) d r\right] \\
& \leq C \mathbb{E}\left[\left(\int _ { S } ^ { T } K ( s , r ) \left(\left|y_{\gamma_{t}}(r-)-\bar{y}_{\gamma_{t}}(r-)\right|+\left\|z_{\gamma_{t}}(r, s)-\bar{z}_{\gamma_{t}}(r, s)\right\|\right.\right.\right. \\
& \left.\left.\left.+\int_{\mathbb{R}^{l}}\left|u_{\gamma_{t}}(r, s, z)-\bar{u}_{\gamma_{t}}(r, s, z)\right| v(d z)\right) d r\right)^{2}\right]
\end{aligned}
$$

a.e. $s \in[S, T]$.

Similar to Eq. (15), we get with Hölder's inequality and $\varepsilon>0$ for $s \in[S, T]$

$$
\begin{aligned}
\| & \left(\Theta^{t, \gamma}\left(y_{\gamma_{t}}(\cdot), z_{\gamma_{t}}(\cdot, \cdot), u_{\gamma_{t}}(\cdot, \cdot, \cdot)\right)-\Theta^{t, \gamma}\left(\bar{y}_{\gamma_{t}}(\cdot), \bar{z}_{\gamma_{t}}(\cdot, \cdot), \bar{u}_{\gamma_{t}}(\cdot, \cdot, \cdot)\right)\right) \|_{\mathbb{M}^{2}[S, T]}^{2} \\
\leq & C(T-S)^{\frac{\varepsilon}{2+\varepsilon}} \mathbb{E}\left[(T-S) \sup _{s \in[S, T]}\left|y_{\gamma_{t}}(s)-\bar{y}_{\gamma_{t}}(s)\right|^{2}+\int_{S}^{T} \int_{S}^{T}\left\|z_{\gamma_{t}}(r, s)-\bar{z}_{\gamma_{t}}(r, s)\right\|^{2} d r d s\right. \\
& \left.+\int_{S}^{T} \int_{S}^{T} \int_{\mathbb{R}^{l}}\left|u_{\gamma_{t}}(r, s, z)-\bar{u}_{\gamma_{t}}(r, s, z)\right|^{2} v(d z) d r d s\right] \\
\leq & C(T-S)^{\frac{\varepsilon}{2+\varepsilon}} \mathbb{E}\left[(T-S) \sup _{s \in[S, T]}\left|y_{\gamma_{t}}(s)-\bar{y}_{\gamma_{t}}(s)\right|^{2}+\int_{S}^{T} \int_{r}^{T}\left\|z_{\gamma_{t}}(r, s)-\bar{z}_{\gamma_{t}}(r, s)\right\|^{2} d s d r\right. \\
& \left.+\int_{S}^{T} \int_{r}^{T} \int_{\mathbb{R}^{l}}\left|u_{\gamma_{t}}(r, s, z)-\bar{u}_{\gamma_{t}}(r, s, z)\right|^{2} v(d z) d s d r\right] \\
\leq & C(T-S)^{\frac{\varepsilon}{2+\varepsilon}}\left\|\left(y_{\gamma_{t}}(\cdot), z_{\gamma_{t}}(\cdot, \cdot), u_{\gamma_{t}}(\cdot, \cdot, \cdot)\right)-\left(\bar{y}_{\gamma_{t}}(\cdot), \bar{z}_{\gamma_{t}}(\cdot, \cdot), \bar{u}_{\gamma_{t}}(\cdot, \cdot, \cdot)\right)\right\|_{\mathbb{M}^{2}[S, T]}^{2} .
\end{aligned}
$$


For $T-S>0$ small enough, the map $\Theta^{t, \gamma}: \mathbb{M}^{2}[S, T] \rightarrow \mathbb{M}^{2}[S, T]$ is a contraction mapping and it admits a unique fixed point $\left(Y_{\gamma_{t}}(\cdot), Z_{\gamma_{t}}(\cdot), U_{\gamma_{t}}(\cdot, \cdot, \cdot)\right) \in \mathbb{M}^{2}[S, T]$ which is the unique adapted M-solution of BSVIE (1) over [S, T]. Moreover, Eq. (14) yields the following estimate

$$
\begin{aligned}
& \mathbb{E}\left[\sup _{s \in[S, T]}\left|Y_{\gamma_{t}}(s)\right|^{2}+\int_{S}^{T} \int_{S}^{T}\left\|Z_{\gamma_{t}}(s, r)\right\|^{2} d r d s+\int_{S}^{T} \int_{S}^{T} \int_{\mathbb{R}^{l}}\left|U_{\gamma_{t}}(s, r, z)\right|^{2} v(d z) d r d s\right] \\
& \leq C\left(\mathbb{E}\left[\int_{S}^{T}\left|\Phi\left(X_{\gamma_{t}, s-}\right)\right|^{2} d s\right]+\mathbb{E}\left[\int_{S}^{T} \int_{S}^{T}\left|f_{0}\left(s, r, X_{\gamma_{t}, r-}\right)\right|^{2} d r d s\right]\right) .
\end{aligned}
$$

Second step: Let $R \in[t, S)$. Now, we want to determine $\left(Z_{\gamma_{t}}(s, r), U_{\gamma_{t}}(s, r, z)\right)$ for $(s, r) \in[S, T] \times[R, S]$ and $z \in \mathbb{R}^{l}$. Since $Y_{\gamma_{t}} \in S^{2}[S, T]$, the process $M(r)=\mathbb{E}\left[Y_{\gamma_{t}}(s) \mid \mathcal{F}_{r}^{t}\right], s \in[S, T]$, is a square integrable martingale and the martingale representation (PR) yields

$\mathbb{E}\left[Y_{\gamma_{t}}(s) \mid \mathcal{F}_{S}^{t}\right]=\mathbb{E}\left[Y_{\gamma_{t}}(s) \mid \mathcal{F}_{R}^{t}\right]+\int_{R}^{S} Z_{\gamma_{t}}(s, r) d W(r)+\int_{R}^{S} \int_{\mathbb{R}^{l}} U_{\gamma_{t}}(s, r, z) \tilde{N}(d r, d z)$.

Furthermore, we obtain with the Burkholder-Davis-Gundy and Doob's inequality

$\mathbb{E}\left[\int_{R}^{S}\left\|Z_{\gamma_{t}}(s, r)\right\|^{2} d r+\int_{R}^{S} \int_{\mathbb{R}^{l}}\left|U_{\gamma_{t}}(s, r, z)\right|^{2} v(d z) d r\right] \leq 4 C \mathbb{E}\left[\left|Y_{\gamma_{t}}(s)\right|^{2}\right]$.

It follows with Eq. (17)

$\mathbb{E}\left[\sup _{s \in[S, T]}\left|Y_{\gamma_{t}}(s)\right|^{2}+\int_{S}^{T} \int_{R}^{S}\left\|Z_{\gamma_{t}}(s, r)\right\|^{2} d r d s+\int_{S}^{T} \int_{R}^{S} \int_{\mathbb{R}^{l}}\left|U_{\gamma_{t}}(s, r, z)\right|^{2} v(d z) d r d s\right]$
$\leq C\left(\mathbb{E}\left[\int_{S}^{T}\left|\Phi\left(X_{\gamma_{t}, s-}\right)\right|^{2} d s\right]+\mathbb{E}\left[\int_{S}^{T} \int_{S}^{T}\left|f_{0}\left(s, r, X_{\gamma_{t}, r-}\right)\right|^{2} d r d s\right]\right)$.

Combining this result and the result of the first step, we get the existence and uniqueness of the adapted M-solution $\left(Y_{\gamma_{t}}(\cdot), Z_{\gamma_{t}}(\cdot, \cdot), U_{\gamma_{t}}(\cdot, \cdot, \cdot)\right)$ to BSVIE (1) on $[S, T] \times[R, T]$. Estimates (17) and (18) provide the following estimate

$$
\begin{aligned}
& \mathbb{E}\left[\sup _{s \in[S, T]}\left|Y_{\gamma_{t}}(s)\right|^{2}+\int_{S}^{T} \int_{R}^{T}\left\|Z_{\gamma_{t}}(s, r)\right\|^{2} d r d s+\int_{S}^{T} \int_{R}^{T} \int_{\mathbb{R}^{l}}\left|U_{\gamma_{t}}(s, r, z)\right|^{2} v(d z) d r d s\right] \\
& \leq C\left(\mathbb{E}\left[\int_{S}^{T}\left|\Phi\left(X_{\gamma_{t}, s-}\right)\right|^{2} d s\right]+\mathbb{E}\left[\int_{S}^{T} \int_{S}^{T}\left|f_{0}\left(s, r, X_{\gamma_{t}, r-}\right)\right|^{2} d r d s\right]\right) .
\end{aligned}
$$

Third step: Now, we consider a stochastic integral equation of the form

$$
\begin{aligned}
\psi_{\gamma_{t}}^{S}(r)= & \Phi\left(X_{\gamma_{t}, r}\right)+\int_{S}^{T} f^{S}\left(r, s, X_{\gamma_{t}, s-}, Z_{\gamma_{t}}(r, s), U_{\gamma_{t}}(r, s)\right) d s \\
& -\int_{S}^{T} Z_{\gamma_{t}}(r, s) d W(s)-\int_{S}^{T} \int_{\mathbb{R}^{l}} U_{\gamma_{t}}(r, s, z) \tilde{N}(d s, d z), \quad r \in[R, S],
\end{aligned}
$$


where we define $f^{S}:[R, S] \times[S, T] \times D\left([0, T], \mathbb{R}^{d}\right) \times \mathbb{R}^{m \times d} \times \mathcal{M}\left(\mathbb{R}^{l}, \mathbb{R}^{m}\right) \rightarrow \mathbb{R}^{m}$ by

$$
f^{S}\left(r, s, X_{\gamma_{t}, s-}, z, u\right)=f\left(r, s, X_{\gamma_{t}, s-}, Y_{\gamma_{t}}(s-), z, Z_{\gamma_{t}}(s, r), u, U_{\gamma_{t}}(s, r)\right) .
$$

Similar techniques to those in Yong (2008) can be used to prove an existence and uniqueness result to solutions

$$
\left(\psi_{\gamma_{t}}^{S}(\cdot), Z_{\gamma_{t}}(\cdot, \cdot), U_{\gamma_{t}}(\cdot, \cdot, \cdot)\right) \in L_{\mathcal{F}_{S}^{t}}^{2}(R, S) \times L^{2}\left(R, S ; M^{2}[S, T]\right) \times L^{2}\left(R, S ; L_{N}^{2}(S, T)\right)
$$

of the stochastic integral equation (19). Thus, $Z_{\gamma_{t}}(r, s)$ and $U_{\gamma_{t}}(r, s, x)$ are unique determined for $(r, s) \in[R, S] \times[S, T], x \in \mathbb{R}^{l}$. For $T-S$ small the following estimate holds

$$
\begin{aligned}
\mathbb{E} & {\left[\left|\psi_{\gamma_{t}}^{S}(r)\right|^{2}+\int_{S}^{T}\left\|Z_{\gamma_{t}}(r, s)\right\|^{2} d s+\int_{S}^{T} \int_{\mathbb{R}^{l}}\left|U_{\gamma_{t}}(r, s, z)\right|^{2} v(d z) d s\right] } \\
\leq & C\left(\mathbb{E}\left[\left|\Phi\left(X_{\gamma_{t}, r}\right)\right|^{2}\right]+\mathbb{E}\left[\left(\int_{S}^{T}\left|f_{0}^{S}\left(r, s, X_{\gamma_{t}, s-}\right)\right| d s\right)^{2}\right]\right) \\
\leq & C\left(\mathbb{E}\left[\left|\Phi\left(X_{\gamma_{t}, r}\right)\right|^{2}\right]+\mathbb{E}\left[\left(\int_{S}^{T}\left|f_{0}\left(r, s, X_{\gamma_{t}, s-}\right)\right| d s\right)^{2}\right]\right. \\
& \left.+(T-S)^{\frac{\varepsilon}{2+\varepsilon}} K \mathbb{E}\right] \sup _{s \in[S, T]}\left|Y_{\gamma_{t}}(s)\right|^{2}+\int_{S}^{T}\left\|Z_{\gamma_{t}}(s, r)\right\|^{2} d s \\
& \left.\left.+\int_{S}^{T} \int_{\mathbb{R}^{l}}\left|U_{\gamma_{t}}(s, r, z)\right|^{2} v(d z) d s\right]\right) \\
\leq & C\left(\mathbb{E}\left[\left|\Phi\left(X_{\gamma_{t}, r}\right)\right|^{2}\right]+\mathbb{E}\left[\left(\int_{S}^{T}\left|f_{0}\left(r, s, X_{\gamma_{t}, s-}\right)\right| d s\right)^{2}\right]\right. \\
& \left.+\mathbb{E}\left[\sup _{s \in[S, T]}\left|Y_{\gamma_{t}}(s)\right|^{2}+\int_{S}^{T}\left\|Z_{\gamma_{t}}(s, r)\right\|^{2} d s+\int_{S}^{T} \int_{\mathbb{R}^{l}}\left|U_{\gamma_{t}}(s, r, z)\right|^{2} v(d z) d s\right]\right),
\end{aligned}
$$

a.e. $r \in[R, S]$.

Together with estimate (18), this yields

$$
\begin{aligned}
& \mathbb{E}\left[\int_{R}^{S}\left|\psi_{\gamma_{t}}^{S}(r-)\right|^{2} d r+\int_{R}^{S} \int_{S}^{T}\left\|Z_{\gamma_{t}}(r, s)\right\|^{2} d s d r+\int_{R}^{S} \int_{S}^{T} \int_{\mathbb{R}^{l}}\left|U_{\gamma_{t}}(r, s, z)\right|^{2} v(d z) d s d r\right] \\
& \leq C\left(\mathbb{E}\left[\int_{S}^{T}\left|\Phi\left(X_{\gamma_{t}, r-}\right)\right|^{2} d r\right]+\mathbb{E}\left[\int_{S}^{T} \int_{S}^{T}\left|f_{0}\left(r, s, X_{\gamma_{t}, s-}\right)\right|^{2} d s d r\right]\right)
\end{aligned}
$$

and for $(r, s) \in[R, S] \times[S, T]$, the processes $Z_{\gamma_{t}}(r, s)$ and $U_{\gamma_{t}}(r, s, x)$ are uniquely determined.

Fourth step: In the last step, we prove that the path-dependent BSVIE (1) admits a unique adapted M-solution on $[R, S]$. For $r \in[R, S]$ we observe a path-dependent BSVIE with jumps of the form 


$$
\begin{aligned}
Y_{\gamma_{t}}(r)= & \psi_{\gamma_{t}}^{S}(r)+\int_{r}^{S} f\left(r, s, X_{\gamma_{t}, s-}, Y_{\gamma_{t}}(s-), Z_{\gamma_{t}}(r, s), Z_{\gamma_{t}}(s, r), U_{\gamma_{t}}(r, s), U_{\gamma_{t}}(s, r)\right) d s \\
& -\int_{r}^{S} Z_{\gamma_{t}}(r, s) d W(s)-\int_{r}^{S} \int_{\mathbb{R}^{l}} U_{\gamma_{t}}(r, s, z) \tilde{N}(d s, d z), \quad r \in[R, S],
\end{aligned}
$$

where $\psi_{\gamma_{t}}^{S}(\cdot) \in L_{\mathcal{F}_{S}^{t}}^{2}(R, S)$. Similarly to the first step, we can show that for $S-R$ small enough BSVIE (21) admits a unique adapted M-solution $\left(Y_{\gamma_{t}}(\cdot), Z_{\gamma_{t}}\right.$ $\left.(\cdot, \cdot), U_{\gamma_{t}}(\cdot, \cdot, \cdot)\right) \in \mathcal{H}^{2}[R, S]$ and it is

$$
\begin{aligned}
& \mathbb{E}\left[\sup _{r \in[R, S]}\left|Y_{\gamma_{t}}(r)\right|^{2}+\int_{R}^{S} \int_{R}^{S}\left\|Z_{\gamma_{t}}(r, s)\right\|^{2} d s d r+\int_{R}^{S} \int_{R}^{S} \int_{\mathbb{R}^{l}}\left|U_{\gamma_{t}}(r, s, z)\right|^{2} v(d z) d s d r\right] \\
& \leq C\left(\mathbb{E}\left[\int_{R}^{S}\left|\Phi\left(X_{\gamma_{t}, r-}\right)\right|^{2} d r\right]+\mathbb{E}\left[\int_{R}^{S} \int_{R}^{S}\left|f_{0}\left(r, s, X_{\gamma_{t}, s-}\right)\right|^{2} d s d r\right]\right) .
\end{aligned}
$$

Insert Eq. (19) to get for $r \in[R, S]$

$$
\begin{aligned}
Y_{\gamma_{t}}(r)= & \psi_{\gamma_{t}}^{S}(r)+\int_{r}^{S} f\left(r, s, X_{\gamma_{t}, s-}, Y_{\gamma_{t}}(s-), Z_{\gamma_{t}}(r, s), Z_{\gamma_{t}}(s, r), U_{\gamma_{t}}(r, s), U_{\gamma_{t}}(s, r)\right) d s \\
& -\int_{r}^{S} Z_{\gamma_{t}}(r, s) d W(s)-\int_{r}^{S} \int_{\mathbb{R}^{l}} U_{\gamma_{t}}(r, s, z) \tilde{N}(d s, d z) \\
= & \Phi\left(X_{\gamma_{t}, r}\right)+\int_{r}^{T} f\left(r, s, X_{\gamma_{t}, s-}, Y_{\gamma_{t}}(s-), Z_{\gamma_{t}}(r, s), Z_{\gamma_{t}}(s, r), U_{\gamma_{t}}(r, s), U_{\gamma_{t}}(s, r)\right) d s \\
& -\int_{r}^{T} Z_{\gamma_{t}}(r, s) d W(s)-\int_{r}^{T} \int_{\mathbb{R}^{l}} U_{\gamma_{t}}(r, s, z) \tilde{N}(d s, d z) .
\end{aligned}
$$

Altogether, we determined the unique adapted M-solution to BSVIE (1) on $[R, T]$ and the following estimate holds

$$
\begin{aligned}
& \mathbb{E}\left[\sup _{r \in[R, T]}\left|Y_{\gamma_{t}}(r)\right|^{2}+\int_{R}^{T} \int_{R}^{T}\left\|Z_{\gamma_{t}}(r, s)\right\|^{2} d s d r+\int_{R}^{T} \int_{R}^{T} \int_{\mathbb{R}^{l}}\left|U_{\gamma_{t}}(r, s, z)\right|^{2} v(d z) d s d r\right] \\
& \leq C \mathbb{E}\left[\int_{R}^{T}\left|\Phi\left(X_{\gamma_{t}, s-}\right)\right| d s+\int_{R}^{T} \int_{R}^{T}\left|f_{0}\left(r, s, X_{\gamma_{t}, s-}\right)\right|^{2} d s d r\right] .
\end{aligned}
$$

If we consider small segments of the rectangle $[t, T]^{2}$ of the form $\left[R_{k}, T\right]^{2}, k=$ $0, \ldots, n, n \in \mathbb{N}, R_{0}=S, R_{1}=R$ and $R_{n}=t$, we get the existence and uniqueness of an adapted M-solution to BSVIE (1) over all rectangles $\left[R_{k}, T\right]^{2}$. We use the same steps as before to show that BSVIE (1) has a unique adapted M-solution over $\left[R_{k}, T\right]$ since we know that BSVIE (1) admits a unique adapted M-solution over $\left[R_{k-1}, T\right]$.

The following result of the stability estimate will be useful for the next section. We consider a path-dependent BSVIE with jumps where the last components of the generator $f$ enter as integrals with respect to Lévy measure $v$ 


$$
\begin{aligned}
& Y_{\gamma_{t}}(s) \\
& =\Phi\left(X_{\gamma_{t}, s}\right)+\int_{s}^{T} f\left(s, r, X_{\gamma_{t}, r-}, Y_{\gamma_{t}}(r-), Z_{\gamma_{t}}(s, r), Z_{\gamma_{t}}(r, s),\right. \\
& \left.\int_{\mathbb{R}^{l}} U_{\gamma_{t}}(s, r, z) \delta(s, r, z) v(d z), \int_{\mathbb{R}^{l}} U_{\gamma_{t}}(r, s, z) \delta(r, s, z) v(d z)\right) d r \\
& \quad-\int_{s}^{T} Z_{\gamma_{t}}(s, r) d W(r)-\int_{s}^{T} \int_{\mathbb{R}^{l}} U_{\gamma_{t}}(s, r, z) \tilde{N}(d r, d z), \quad s \in[t, T],
\end{aligned}
$$

where we assume

(H'1) $\Phi \in L_{\mathcal{F}_{T}^{t}}^{2}\left(D\left([0, T], \mathbb{R}^{d}\right) ; \mathbb{R}^{m}\right)$.

$f: \Omega \times \Delta^{c}[t, T] \times D\left([0, T], \mathbb{R}^{d}\right) \times \mathbb{R}^{m} \times \mathbb{R}^{m \times d} \times \mathbb{R}^{m \times d} \times \mathbb{R}^{m} \times \mathbb{R}^{m} \rightarrow \mathbb{R}^{m}$

is $\quad \mathcal{F}_{T}^{t} \otimes \mathcal{B}\left(\Delta^{c}[t, T] \times D\left([0, T], \mathbb{R}^{d}\right) \times \mathbb{R}^{m} \times \mathbb{R}^{m \times d} \times \mathbb{R}^{m \times d} \times \mathbb{R}^{m} \times \mathbb{R}^{m}\right)$ measurable such that

$$
r \mapsto f(s, r, \gamma, y, z, \zeta, u, \xi)
$$

is $\mathbb{F}$-progressive measurable. Furthermore, generator $f$ is Lipschitz continuous in the sense that

$$
\begin{aligned}
& |f(\omega, s, r, \gamma, y, z, \zeta, u, \xi)-f(\omega, s, r, \bar{\gamma}, \bar{y}, \bar{z}, \bar{\zeta}, \bar{u}, \bar{\xi})| \\
& \leq K(s, r)\left(\|\gamma-\bar{\gamma}\|_{D}+|y-\bar{y}|+\|z-\bar{z}\|+\|\zeta-\bar{\zeta}\|\right. \\
& \quad+|u(x)-\bar{u}(x)|+|\xi(x)-\bar{\xi}(x)|),
\end{aligned}
$$

a.e. $(\omega, s, r) \in \Omega \times \Delta^{c}[t, T]$ for all $(\gamma, y, z, \zeta, u, \xi),(\bar{\gamma}, \bar{y}, \bar{z}, \bar{\zeta}, \bar{u}, \bar{\xi}) \quad \in$ $D\left([0, T], \mathbb{R}^{d}\right) \times \mathbb{R}^{m} \times \mathbb{R}^{m \times d} \times \mathbb{R}^{m \times d} \times \mathbb{R}^{m} \times \mathbb{R}^{m} . K: \Delta^{c} \rightarrow[0, \infty)$ is a deterministic function such that for $\varepsilon>0$,

$$
\sup _{s \in[t, T]} \int_{s}^{T}|K(s, r)|^{2+\varepsilon} d r<\infty .
$$

(H'3) $\mathbb{E}\left[\int_{t}^{T} \int_{s}^{T}\left|f_{0}\left(s, r, \gamma_{r-}\right)\right|^{2} d r d s\right]<\infty$, where $f_{0}\left(s, r, \gamma_{r-}\right)=f\left(s, r, \gamma_{r-}\right.$, $0,0,0,0,0)$.

(H'4) $\quad \delta: \Omega \times[t, T]^{2} \times \mathbb{R}^{l} \rightarrow \mathbb{R}^{m}$ is measurable such that for any $(s, r) \in[t, T]^{2}$

$$
\int_{\mathbb{R}^{l}}|\delta(s, r, z)|^{2} v(d z)<\infty .
$$

Corollary 2 Let assumptions (H'l)-(H'4) hold and $\left(Y_{\gamma_{t}}(\cdot), Z_{\gamma_{t}}(\cdot, \cdot)\right.$, $\left.U_{\gamma_{t}}(\cdot, \cdot, \cdot)\right) \in \mathcal{H}^{2}[t, T]$ and $\left(\bar{Y}_{\gamma_{t}}(\cdot), \bar{Z}_{\gamma_{t}}(\cdot, \cdot), \bar{U}_{\gamma_{t}}(\cdot, \cdot, \cdot)\right) \in \mathcal{H}^{2}[t, T]$ be the unique adapted M-solutions to BSVIE (23) with respect to $(f, \Phi)$ and $(\bar{f}, \bar{\Phi})$, respectively. Then, for any $\gamma \in D\left([0, T], \mathbb{R}^{d}\right)$, the following stability estimate holds 


$$
\begin{aligned}
& \left\|\left(Y_{\gamma_{t}}(\cdot), Z_{\gamma_{t}}(\cdot, \cdot), U_{\gamma_{t}}(\cdot, \cdot, \cdot)\right)-\left(\bar{Y}_{\gamma_{t}}(\cdot), \bar{Z}_{\gamma_{t}}(\cdot, \cdot), \bar{U}_{\gamma_{t}}(\cdot, \cdot, \cdot)\right)\right\|_{\mathcal{H}^{2}[S, T]}^{2} \\
& \leq C \mathbb{E}\left[\int_{S}^{T}\left|\Phi\left(X_{\gamma_{t}, s-}\right)-\bar{\Phi}\left(X_{\gamma_{t}, s-}\right)\right|^{2} d s+\int_{S}^{T} \int_{S}^{T} \mid f\left(s, r, X_{\gamma_{t}, r-}, Y_{\gamma_{t}}(r-), Z_{\gamma_{t}}(s, r),\right.\right. \\
& \left.\quad Z_{\gamma_{t}}(r, s), \int_{\mathbb{R}^{l}} U_{\gamma_{t}}(s, r, z) \delta(s, r, z) v(d z), \int_{\mathbb{R}^{l}} U_{\gamma_{t}}(r, s, z) \delta(r, s, z) v(d z)\right) \\
& \quad-\bar{f}\left(s, r, X_{\gamma_{t}, r-}, Y_{\gamma_{t}}(r-), Z_{\gamma_{t}}(s, r), Z_{\gamma_{t}}(r, s), \int_{\mathbb{R}^{l}} U_{\gamma_{t}}(s, r, z) \delta(s, r, z) v(d z),\right. \\
& \left.\left.\quad \int_{\mathbb{R}^{l}} U_{\gamma_{t}}(r, s, z) \delta(r, s, z) v(d z)\right)\left.\right|^{2} d r d s\right] .
\end{aligned}
$$

Proof This proof follows the techniques in Yong (2008). We define for $(s, r) \in \Delta^{c}$ and $z \in \mathbb{R}^{l}$

$$
\begin{array}{ll}
\hat{\Phi}\left(X_{\gamma_{t}, s}\right)=\Phi\left(X_{\gamma_{t}, s}\right)-\bar{\Phi}\left(X_{\bar{\gamma}_{t}, s}\right), & \hat{Y}_{\gamma_{t}}(s)=Y_{\gamma_{t}}(s)-\bar{Y}_{\bar{\gamma}_{t}}(s), \\
\hat{Z}_{\gamma_{t}}(s, r)=Z_{\gamma_{t}}(s, r)-\bar{Z}_{\bar{\gamma}_{t}}(s, r), & \hat{U}_{\gamma_{t}}(s, r, z)=U_{\gamma_{t}}(s, r, z)-\bar{U}_{\bar{\gamma}_{t}}(s, r, z),
\end{array}
$$

and

$$
\begin{aligned}
& \hat{f}\left(s, r, X_{\gamma_{t}, r-}\right) \\
& =f\left(s, r, X_{\gamma_{t}, r-}, Y_{\gamma_{t}}(r-), Z_{\gamma_{t}}(s, r), Z_{\gamma_{t}}(r, s),\right. \\
& \left.\int_{\mathbb{R}^{l}} U_{\gamma_{t}}(s, r, z) \delta(s, r, z) v(d z), \int_{\mathbb{R}^{l}} U_{\gamma_{t}}(r, s, z) \delta(r, s, z) v(d z)\right) \\
& -\bar{f}\left(s, r, X_{\gamma_{t}, r-}, Y_{\gamma_{t}}(r-), Z_{\gamma_{t}}(s, r), Z_{\gamma_{t}}(r, s),\right. \\
& \left.\int_{\mathbb{R}^{l}} U_{\gamma_{t}}(s, r, z) \delta(s, r, z) v(d z), \int_{\mathbb{R}^{l}} U_{\gamma_{t}}(r, s, z) \delta(r, s, z) v(d z)\right), \\
& \bar{f}_{z_{i}}\left(s, r, X_{\gamma_{t}, r-}\right) \\
& =\left(\overline { f } \left(s, r, X_{\gamma_{t}, r-}, \bar{Y}_{\gamma_{t}}(r-), Z_{\gamma_{t}}(s, r), Z_{\gamma_{t}}(r, s)\right.\right. \text {, } \\
& \left.\int_{\mathbb{R}^{l}} U_{\gamma_{t}}(s, r, z) \delta(s, r, z) v(d z), \int_{\mathbb{R}^{l}} U_{\gamma_{t}}(r, s, z) \delta(r, s, z) v(d z)\right) \\
& -\bar{f}\left(s, r, X_{\gamma_{t}, r-}, \bar{Y}_{\gamma_{t}}(r-), \bar{Z}_{\gamma_{t}}^{i}(s, r), Z_{\gamma_{t}}(r, s),\right. \\
& \left.\left.\int_{\mathbb{R}^{l}} U_{\gamma_{t}}(s, r, z) \delta(s, r, z) v(d z), \int_{\mathbb{R}^{l}} U_{\gamma_{t}}(r, s, z) \delta(r, s, z) v(d z)\right)\right) \\
& \frac{\hat{Z}_{\gamma_{t}}^{i}(s, r)^{T}}{\left|\hat{Z}_{\gamma_{t}}^{i}(s, r)\right|^{2}} \mathbb{1}_{\left\{\hat{Z}_{\gamma_{t}}^{i}(s, r) \neq 0\right\}},
\end{aligned}
$$

where $\bar{Z}_{\gamma_{t}}^{i}(s, r)=\left(\bar{Z}_{\gamma_{t}}^{1}(s, r), \ldots, \bar{Z}_{\gamma_{t}}^{i}(s, r), Z_{\gamma_{t}}^{i+1}(s, r), \ldots, Z_{\gamma_{t}}^{d}(s, r)\right), i=$ $1, \ldots, d . \bar{f}_{y}\left(s, r, X_{\gamma_{t}, r-}\right), \bar{f}_{\zeta_{i}}\left(s, r, X_{\gamma_{t}, r-}\right), \bar{f}_{u}\left(s, r, X_{\gamma_{t}, r-}\right)$ and $\bar{f}_{\xi}\left(s, r, X_{\gamma_{t} r-}\right)$ are defined quite similarly.

Since $\left(Y_{\gamma_{t}}(\cdot), Z_{\gamma_{t}}(\cdot, \cdot), U_{\gamma_{t}}(\cdot, \cdot, \cdot)\right),\left(\bar{Y}_{\bar{\gamma}_{t}}(\cdot), \bar{Z}_{\bar{\gamma}_{t}}(\cdot, \cdot), \bar{U}_{\bar{\gamma}_{t}}(\cdot, \cdot, \cdot)\right) \in \mathcal{H}^{2}[t, T]$ are unique adapted M-solutions to $\operatorname{BSVIE}(1)$ with respect to $(f, \Phi)$ and $(\bar{f}, \bar{\Phi})$, respectively, we get 


$$
\begin{aligned}
& \hat{Y}_{\gamma_{t}}(s) \\
& =\hat{\Phi}\left(X_{\gamma_{t}, s}\right)+\int_{s}^{T}\left[\hat{f}\left(s, r, X_{\gamma_{t}, r-}\right)+\bar{f}_{y}\left(s, r, X_{\gamma_{t}, r-}\right) \hat{Y}_{\gamma_{t}}(r-)+\sum_{i=1}^{d} \bar{f}_{z_{i}}\left(s, r, X_{\gamma_{t}, r-}\right) \hat{Z}_{\gamma_{t}}^{i}(s, r)\right. \\
& \quad+\sum_{i=1}^{d} \bar{f}_{\zeta_{i}}\left(s, r, X_{\gamma_{t}, r-}\right) \cdot \hat{Z}_{\gamma_{t}}^{i}(r, s)+\bar{f}_{u}\left(s, r, X_{\gamma_{t}, r-}\right) \cdot \int_{\mathbb{R}^{l}} U_{\gamma_{t}}(s, r, z) \delta(s, r, z) v(d z) \\
& \left.\quad+\bar{f}_{\xi}\left(s, r, X_{\gamma_{t}, r-}\right) \cdot \int_{\mathbb{R}^{l}} U_{\gamma_{t}}(r, s, z) \delta(r, s, z) v(d z)\right] d r \\
& \quad-\int_{s}^{T} \hat{Z}_{\gamma_{t}}(s, r) d W(r)-\int_{s}^{T} \int_{\mathbb{R}^{l}} \hat{U}_{\gamma_{t}}(s, r, z) \tilde{N}(d r, d z) .
\end{aligned}
$$

Theorem 2 yields that $\left(\hat{Y}_{\gamma_{t}}(\cdot), \hat{Z}_{\gamma_{t}}(\cdot, \cdot), \hat{U}_{\gamma_{t}}(\cdot, \cdot, \cdot)\right)$ is the unique adapted M-solution to BSVIE (25) and the following estimate holds

$$
\begin{aligned}
& \left\|\left(\hat{Y}_{\gamma_{t}}(\cdot), \hat{Z}_{\gamma_{t}}(\cdot, \cdot), \hat{U}_{\gamma_{t}}(\cdot, \cdot, \cdot)\right)\right\|_{\mathcal{H}[t, T]}^{2} \\
& \leq C \mathbb{E}\left[\int_{t}^{T}\left|\hat{\Phi}\left(X_{\gamma_{t}, s-}\right)\right|^{2} d s+\int_{t}^{T} \int_{t}^{T}\left|\hat{f}\left(s, r, X_{\gamma_{t}, r-}\right)\right|^{2} d r d s\right],
\end{aligned}
$$

which provides the stability estimate.

\section{Corollary 3 Consider a BSVIE of the form}

$$
\begin{aligned}
& Y_{\gamma_{t}}(s) \\
& =\Phi\left(X_{\gamma_{t}, s}\right)+\int_{s}^{T}\left[m \left(s, r, X_{\gamma_{t}, r-}, Y_{\gamma_{t}}(r-), Z_{\gamma_{t}}(s, r), Z_{\gamma_{t}}(r, s),\right.\right. \\
& \left.\left.\quad \int_{\mathbb{R}^{l}} U_{\gamma_{t}}(s, r, z) \delta(s, r, z) v(d z), \int_{\mathbb{R}^{l}} U_{\gamma_{t}}(r, s, z) \delta(r, s, z) v(d z)\right)+A\left(s, r, X_{\gamma_{t}, r-}\right)\right] d r \\
& \quad-\int_{s}^{T} Z_{\gamma_{t}}(s, r) d W(r)-\int_{s}^{T} \int_{\mathbb{R}^{l}} U_{\gamma_{t}}(s, r, z) \tilde{N}(d r, d z), \quad s \in[t, T],
\end{aligned}
$$

where $\delta$ satisfies (H'4). Furthermore, let

$$
m: \Omega \times \Delta^{c}[t, T] \times D\left([0, T], \mathbb{R}^{d}\right) \times \mathbb{R}^{m} \times \mathbb{R}^{m \times d} \times \mathbb{R}^{m \times d} \times \mathbb{R}^{m} \times \mathbb{R}^{m} \rightarrow \mathbb{R}^{m}
$$

be $\mathcal{F}_{T}^{t} \otimes \mathcal{B}\left(\Delta^{c}[t, T] \times D\left([0, T], \mathbb{R}^{d}\right) \times \mathbb{R}^{m} \times \mathbb{R}^{m \times d} \times \mathbb{R}^{m \times d} \times \mathbb{R}^{m} \times \mathbb{R}^{m}\right)$ measurable such that

$$
r \mapsto m(s, r, \gamma, y, z, \zeta, u, \xi)
$$

is $\mathbb{F}$-progressive measurable.

Moreover, let $m$ be linear in $(y, z, \zeta, u, \xi)$ such that $m(s, r, \gamma, 0,0,0,0,0)=0$ a.s. for all $\gamma \in D\left([0, T], \mathbb{R}^{d}\right),(s, r) \in \Delta^{c}[t, T]$, and

$$
|m(\omega, s, r, \gamma, y, z, \zeta, u, \xi)| \leq K(s, r)(|y|+\|z\|+\|\zeta\|+|u|+|\xi|),
$$

a.e. $(\omega, s, r) \in \Omega \times \Delta^{c}[t, T]$ for all $(\gamma, y, z, \zeta, u, \xi) \in D\left([0, T], \mathbb{R}^{d}\right) \times \mathbb{R}^{m} \times \mathbb{R}^{m \times d} \times$ $\mathbb{R}^{m \times d} \times \mathbb{R}^{m} \times \mathbb{R}^{m} . K: \Delta^{c}[t, T] \rightarrow[0, \infty)$ is a deterministic function such that for $\varepsilon>0$ 


$$
\sup _{s \in[t, T]} \int_{s}^{T}|K(s, r)|^{2+\varepsilon} d r<\infty .
$$

$A: \Omega \times \Delta^{c}[t, T] \times D\left([0, T], \mathbb{R}^{d}\right) \rightarrow \mathbb{R}^{m}$ is $\mathcal{F}_{T}^{t} \otimes \mathcal{B}\left(\Delta^{c}[t, T] \times D\left([0, T], \mathbb{R}^{d}\right)\right)-$ measurable such that $r \mapsto A\left(s, r, \gamma_{r-}\right)$ is $\mathbb{F}$-progressive measurable and $\mathbb{E}\left[\int_{t}^{T} \int_{s}^{T}\left|A\left(s, r, \gamma_{r-}\right)\right|^{2} d r d s\right]<\infty$. Then, for all $\gamma \in D\left([0, T], \mathbb{R}^{d}\right)$, BSVIE (26) admits a unique adapted $M$-solution $\left(Y_{\gamma_{t}}(\cdot), Z_{\gamma_{t}}(\cdot, \cdot), U_{\gamma_{t}}(\cdot, \cdot, \cdot)\right) \in \mathcal{H}^{2}[t, T]$ and the following estimate holds

$$
\begin{aligned}
& \left\|\left(Y_{\gamma_{t}}(\cdot), Z_{\gamma_{t}}(\cdot, \cdot), U_{\gamma_{t}}(\cdot, \cdot, \cdot)\right)\right\|_{\mathcal{H}^{2}[t, T]}^{2} \\
& \leq C\left(\mathbb{E}\left[\int_{t}^{T}\left|\Phi\left(X_{\gamma_{t}, s-}\right)\right|^{2} d s\right]+\mathbb{E}\left[\int_{t}^{T} \int_{t}^{T}\left|A\left(s, r, X_{\gamma_{t}, r-}\right)\right|^{2} d r d s\right]\right) .
\end{aligned}
$$

If $\left(Y_{\gamma_{t}}(\cdot), Z_{\gamma_{t}}(\cdot, \cdot), U_{\gamma_{t}}(\cdot, \cdot, \cdot)\right),\left(\bar{Y}_{\gamma_{t}}(\cdot), \bar{Z}_{\gamma_{t}}(\cdot, \cdot), \bar{U}_{\gamma_{t}}(\cdot, \cdot, \cdot)\right) \in \mathcal{H}^{2}[t, T]$ are the unique adapted $M$-solution to BSVIE (23) with respect to $(m, A, \Phi)$ and $(\bar{m}, \bar{A}, \bar{\Phi})$, respectively, the following stability estimate holds

$$
\begin{aligned}
& \left\|\left(Y_{\gamma_{t}}(\cdot), Z_{\gamma_{t}}(\cdot, \cdot), U_{\gamma_{t}}(\cdot, \cdot, \cdot)\right)-\left(\bar{Y}_{\gamma_{t}}(\cdot), \bar{Z}_{\gamma_{t}}(\cdot, \cdot), \bar{U}_{\gamma_{t}}(\cdot, \cdot, \cdot)\right)\right\|_{\mathcal{H}^{2}[S, T]}^{2} \\
& \leq C\left(\mathbb{E}\left[\int_{S}^{T}\left|\Phi\left(X_{\gamma_{t}, s-}\right)-\bar{\Phi}\left(X_{\gamma_{t}, s-}\right)\right|^{2} d s\right]\right. \\
& \left.\quad+\mathbb{E}\left[\int_{S}^{T} \int_{S}^{T}\left|A\left(s, r, X_{\gamma_{t}, r-}\right)-\bar{A}\left(s, r, X_{\gamma_{t}, r-}\right)\right|^{2} d r d s\right]\right)
\end{aligned}
$$

for any $S \in[t, T)$.

Proof This corollary is a consequence of the results in Theorem 2 and Corollary 2.

\section{Path-differentiability of path-dependent BSVIEs with jumps}

In this section, we consider path-dependent BSVIEs with jumps of the form (23). To prove path-differentiability in the initial path of solutions to path-dependent BSVIEs, we need the following assumptions.

(D1) $\Phi \in L_{\mathcal{F}_{T}^{t}}^{2}\left(D\left([0, T], \mathbb{R}^{d}\right) ; \mathbb{R}^{m}\right)$ is continuous and for $i=1, \ldots, d$ $D_{i}^{1} \Phi(\gamma ;[t, T]) \in L_{\mathcal{F}_{T}^{t}}^{2}\left(D\left([0, T], \mathbb{R}^{d}\right) ; \mathbb{R}^{m}\right)$ exist.

(D2) The generator

$$
\begin{aligned}
& f: \Omega \times \Delta^{c}[t, T] \times D\left([0, T], \mathbb{R}^{d}\right) \times \mathbb{R}^{m} \times \mathbb{R}^{m \times d} \times \mathbb{R}^{m \times d} \times \mathbb{R}^{m} \times \mathbb{R}^{m} \rightarrow \mathbb{R}^{m} \\
& \text { is } \mathcal{F}_{T}^{t} \otimes \mathcal{B}\left(\Delta^{c}[t, T] \times D\left([0, T], \mathbb{R}^{d}\right) \times \mathbb{R}^{m} \times \mathbb{R}^{m \times d} \times \mathbb{R}^{m \times d} \times \mathbb{R}^{m} \times \mathbb{R}^{m}\right) \text { - } \\
& \text { measurable such that }
\end{aligned}
$$

$$
r \mapsto f(s, r, \gamma, y, z, \zeta, u, \xi)
$$


is $\mathbb{F}$-progressive measurable. Furthermore, $(s, r) \mapsto f(s, r, \gamma, y, z, \zeta, u, \xi)$ is Lipschitz continuous in the sense that

$$
\begin{aligned}
& |f(s, r, \gamma, y, z, \zeta, u, \xi)-f(s, r, \bar{\gamma}, \bar{y}, \bar{z}, \bar{\zeta}, \bar{u}, \bar{\xi})| \\
& \leq K(s, r)\left(\|\gamma-\bar{\gamma}\|_{D}+|y-\bar{y}|+\|z-\bar{z}\|+\|\zeta-\bar{\zeta}\|+|u-\bar{u}|+|\xi-\bar{\xi}|\right),
\end{aligned}
$$

a.e. $(\omega, s, r) \in \Omega \times \Delta^{c}[t, T]$, for all $(\gamma, y, z, \zeta, u, \xi),(\bar{\gamma}, \bar{y}, \bar{z}, \bar{\zeta}, \bar{u}, \bar{\xi}) \in$ $D\left([0, T], \mathbb{R}^{d}\right) \times \mathbb{R}^{m} \times \mathbb{R}^{m \times d} \times \mathbb{R}^{m \times d} \times \mathbb{R}^{m} \times \mathbb{R}^{m}$.

Moreover, $K: \Delta^{c}[t, T] \rightarrow[0, \infty)$ is a deterministic function such that for $\varepsilon>0$

$$
\sup _{s \in[t, T]} \int_{s}^{T}|K(s, r)|^{2+\varepsilon} d r<\infty
$$

$$
\mathbb{E}\left[\int_{t}^{T} \int_{t}^{T}\left|f_{0}\left(s, r, \gamma_{r-}\right)\right|^{2} d r\right]<\infty
$$

(D4) For all $\bar{\gamma} \in D\left([0, T], \mathbb{R}^{d}\right),(s, r) \in \Delta^{c}[t, T]$, the map

$$
(x, y, z, \zeta, u, \xi) \mapsto f\left(s, r, \bar{\gamma}_{r}+x \mathbb{1}_{[t, T]}, y, z, \zeta, u, \xi\right)
$$

is of class $C^{1}\left(\mathbb{R}^{d} \times \mathbb{R}^{m} \times \mathbb{R}^{m \times d} \times \mathbb{R}^{m \times d} \times \mathbb{R}^{m} \times \mathbb{R}^{m} ; \mathbb{R}^{m}\right)$ and the first-order partial derivatives with respect to $(x, y, z, \zeta, u, \xi)$ are uniformly bounded.

(D5) $\delta: \Omega \times[t, T]^{2} \times \mathbb{R}^{l} \rightarrow \mathbb{R}^{m}$ is measurable such that for any $(s, r) \in[t, T]^{2}$

$$
\int_{\mathbb{R}^{l}}|\delta(s, r, z)|^{2} v(d z)<\infty
$$

(D6) Let $\gamma \mapsto U_{\gamma_{t}}(s, r, x)$ be differentiable for all $(s, r, x) \in[t, T]^{2} \times \mathbb{R}^{l}$ and assume that

$$
\int_{\mathbb{R}^{l}}\left|D_{i}^{1} U_{\gamma_{t}}(s, r, x ;[t, T])\right| v(d x)<\infty
$$

for each $(s, r) \in[t, T]^{2}$. Furthermore, assume that there exists a neighborhood $(-\varepsilon, \varepsilon)$ and a process $M_{\gamma_{t}}$ such that for each $(s, r) \in[t, T]^{2}$ $\int_{\mathbb{R}^{l}}\left|M_{\gamma_{t}}(s, r, x)\right| v(d x)<\infty$ and such that for $i=1, \ldots, d$

$$
\frac{\left|U_{\gamma_{t}+h \mathbb{1}_{[t, T]} e_{i}}(s, r, x)-U_{\gamma_{t}}(s, r, x)\right|}{h} \leq M_{\gamma_{t}}^{i}(s, r, x), \quad h \in(-\varepsilon, \varepsilon) .
$$

Theorem 3 Let (D1)-(D6) hold. Then, the function

$$
D\left([0, T], \mathbb{R}^{d}\right) \rightarrow \mathcal{H}^{2}[t, T], \quad \gamma_{t} \mapsto\left(Y_{\gamma_{t}}(\cdot), Z_{\gamma_{t}}(\cdot, \cdot), U_{\gamma_{t}}(\cdot, \cdot, \cdot)\right)
$$

is differentiable in the sense of Definition 1 , where $\mathbb{R}^{m}$ is replaced by the space $\mathcal{H}^{2}[t, T]$. For $i=1, \ldots, d, s \in[t, T]$, the derivative is the unique adapted $M$-solution to a path-dependent BSVIE with jumps of the form 


$$
\begin{aligned}
& D_{i}^{1} Y_{\gamma_{t}}(s ;[t, T]) \\
& =D_{i}^{1} \Phi\left(X_{\gamma_{t}, s} ;[t, T]\right)+\int_{s}^{T} D_{i}^{1} f\left(s, r, X_{\gamma_{t}, r-}, Y_{\gamma_{t}}(r-), Z_{\gamma_{t}}(s, r), Z_{\gamma_{t}}(r, s),\right. \\
& \left.\quad \int_{\mathbb{R}^{l}} U_{\gamma_{t}}(s, r, x) \delta(s, r, x) v(d x), \int_{\mathbb{R}^{l}} U_{\gamma_{t}}(r, s, x) \delta(r, s, x) v(d x) ;[t, T]\right) d r \\
& \quad+\int_{s}^{T}\left[f _ { y } \left(s, r, X_{\gamma_{t}, r-}, Y_{\gamma_{t}}(r-), Z_{\gamma_{t}}(s, r), Z_{\gamma_{t}}(r, s), \int_{\mathbb{R}^{l}} U_{\gamma_{t}}(s, r, x) \delta(s, r, x) v(d x),\right.\right. \\
& \left.\quad \int_{\mathbb{R}^{l}} U_{\gamma_{t}}(r, s, x) \delta(r, s, x) v(d x)\right) \cdot D_{i}^{1} Y_{\gamma_{t}}(r ;[t ; T]) \\
& \quad+\sum_{j=1}^{d} f_{z_{j}}\left(s, r, X_{\gamma_{t}, r-}, Y_{\gamma_{t}}(r-), Z_{\gamma_{t}}(s, r), Z_{\gamma_{t}}(r, s), \int_{\mathbb{R}^{l}} U_{\gamma_{t}}(s, r, x) \delta(s, r, x) v(d x),\right. \\
& \left.\quad \int_{\mathbb{R}^{l}} U_{\gamma_{t}}(r, s, x) \delta(r, s, x) v(d x)\right) \cdot D_{i}^{1} Z_{\gamma_{t}}^{j}(s, r ;[t, T]) \\
& \quad+\sum_{j=1}^{d} f_{\zeta_{j}}\left(s, r, X_{\gamma_{t}, r-}, Y_{\gamma_{t}}(r-), Z_{\gamma_{t}}(s, r), Z_{\gamma_{t}}(r, s), \int_{\mathbb{R}^{l}} U_{\gamma_{t}}(s, r, x) \delta(s, r, x) v(d x),\right. \\
& \left.\left.\quad \int_{\mathbb{R}^{l}} U_{\gamma_{t}}(r, s, x) \delta(r, s, x) v(d x)\right) \cdot D_{i}^{1} U_{\gamma_{t}}(r, s, x ;[t, T]) v(d x)\right] d r \\
& \left.\quad \int_{\mathbb{R}^{l}}^{T} U_{\gamma_{t}}(r, s, x) \delta(r, s, x) v(d x)\right) \cdot D_{i}^{1} Z_{\gamma_{t}}^{j}(r, s ;[t, T]) \\
& \quad+\int_{\mathbb{R}^{l}} f_{u}^{1} Z_{\gamma_{t}}(s, r ;[t, T]) d W(r)-\int_{s}^{T} \int_{\mathbb{R}^{l}} D_{i}^{1} U_{\gamma_{t}}(s, r, x ;[t, T]) \tilde{N}(d r, d x), \\
& \left.\quad \int_{\mathbb{R}^{l}} U_{\gamma_{t}}(r, s, x) \delta(r, s, x) v(d x)\right) \cdot D_{i}^{1} U_{\gamma_{t}}(s, r, x ;[t, T]) v(d x) d r \\
& \quad+\int_{\xi}\left(s, r, X_{\gamma_{t}, r-}, Y_{\gamma_{t}}(r-), Z_{\gamma_{t}}(s, r), Z_{\gamma_{t}}(r, s), \int_{\mathbb{R}^{l}} U_{\gamma_{t}}(s, r, x) \delta(s, r, x) v(d x),\right. \\
& \quad Z_{\gamma_{t}}(s, r), Z_{\gamma_{t}}(r, s), \int_{\mathbb{R}^{l}} U_{\gamma_{t}}(s, r, x) \delta(s, r, x) v(d x),
\end{aligned}
$$

Proof To simplify the notation, we restrict ourselves to the one-dimensional case, $m=d=l=1$. The extension to the multidimensional case is straightforward. We define for $(s, r) \in[t, T]^{2}$ and $x \in \mathbb{R}$

$$
\begin{gathered}
\mathcal{Y}^{h}(s)=\frac{1}{h}\left(Y_{\gamma_{t}^{h}}(s)-Y_{\gamma_{t}}(s)\right), \quad \mathcal{Z}^{h}(s, r)=\frac{1}{h}\left(Z_{\gamma_{t}^{h}}(s, r)-Z_{\gamma_{t}}(s, r)\right), \\
\mathcal{U}^{h}(s, r, x)=\frac{1}{h}\left(U_{\gamma_{t}^{h}}(s, r, x)-U_{\gamma_{t}}(s, r, x)\right), \Delta \Phi^{h}\left(X_{\gamma_{t}, s}\right)=\frac{1}{h}\left(\Phi\left(X_{\gamma_{t}^{h}, s}\right)-\Phi\left(X_{\gamma_{t}, s}\right)\right) .
\end{gathered}
$$

With the definition of $X_{\gamma_{t}, s}$, we get $X_{\gamma_{t}{ }^{h}, s}(u)-X_{\gamma_{t}, s}(u)=h \mathbb{1}_{[t, T]}(u), u \in$ $[0, T], s \in[t, T]$. 
Define for any $(s, r) \in[t, T]^{2}, h \in \mathbb{R} \backslash\{0\}$, the mapping $l_{h}^{s, r}:[0,1] \rightarrow$ $D([0, T], \mathbb{R}) \times \mathbb{R} \times \mathbb{R} \times \mathbb{R} \times \mathbb{R} \times \mathbb{R}$ by

$$
\begin{aligned}
& l_{h}^{s, r}(\theta) \\
&=\left(X_{\gamma_{t}, r-}+\theta\left(X_{\gamma_{t}^{h}, r-}-X_{\gamma_{t}, r-}\right), Y_{\gamma_{t}}(r-)+\theta h \mathcal{Y}^{h}(r-), Z_{\gamma_{t}}(s, r)+\theta h \mathcal{Z}^{h}(s, r),\right. \\
& Z_{\gamma_{t}}(r, s)+\theta h \mathcal{Z}^{h}(r, s), \int_{\mathbb{R}}\left(U_{\gamma_{t}}(s, r, x)+\theta h \mathcal{U}^{h}(s, r, x)\right) \delta(s, r, x) v(d x), \\
&\left.\int_{\mathbb{R}}\left(U_{\gamma_{t}}(r, s, x)+\theta h \mathcal{U}^{h}(r, s, x)\right) \delta(r, s, x) v(d x)\right) \\
&=\left(X_{\gamma_{t}, r-}+\theta h, Y_{\gamma_{t}}(r-)+\theta h \mathcal{Y}^{h}(r-), Z_{\gamma_{t}}(s, r)+\theta h \mathcal{Z}^{h}(s, r),\right. \\
& Z_{\gamma_{t}}(r, s)+\theta h \mathcal{Z}^{h}(r, s), \int_{\mathbb{R}}\left(U_{\gamma_{t}}(s, r, x)+\theta h \mathcal{U}^{h}(s, r, x)\right) \delta(s, r, x) v(d x), \\
&\left.\left.\int_{\mathbb{R}}\left(U_{\gamma_{t}}(r, s, x)+\theta h \mathcal{U}^{h}(r, s, x)\right) \delta(r, s, x) v(d x)\right)\right) .
\end{aligned}
$$

It follows for $(s, r) \in[t, T]^{2}$

$$
\begin{aligned}
\frac{1}{h} \frac{\partial}{\partial \theta} l_{h}^{s, r}(\theta)= & \left(1, \mathcal{Y}^{h}(r-), \mathcal{Z}^{h}(s, r), \mathcal{Z}^{h}(r, s), \int_{\mathbb{R}} \mathcal{U}^{h}(s, r, x) \delta(s, r, x) v(d x),\right. \\
& \left.\int_{\mathbb{R}} \mathcal{U}^{h}(r, s, x) \delta(r, s, x) v(d x)\right)
\end{aligned}
$$

and Corollary 3 provides that for $h \in \mathbb{R} \backslash\{0\}\left(\mathcal{Y}^{h}(\cdot), \mathcal{Z}^{h}(\cdot, \cdot), \mathcal{U}^{h}(\cdot, \cdot, \cdot)\right)$ is the unique adapted M-solution to the linearized path-dependent BSVIE with jumps

$$
\begin{aligned}
& \mathcal{Y}^{h}(s) \\
& =\Delta \Phi^{h}\left(X_{\gamma_{t}, s}\right)+\int_{s}^{T}\left[m _ { h } ^ { s , r } \left(\mathcal{Y}^{h}(r-), \mathcal{Z}^{h}(s, r), \mathcal{Z}^{h}(r, s), \int_{\mathbb{R}} \mathcal{U}^{h}(s, r, x) \delta(s, r, x) v(d x),\right.\right. \\
& \left.\left.\quad \int_{\mathbb{R}} \mathcal{U}^{h}(r, s, x) \delta(r, s, x) v(d x)\right)+A_{h}^{1}(s, r)\right] d r \\
& \quad-\int_{s}^{T} \mathcal{Z}^{h}(s, r) d W(r)-\int_{s}^{T} \int_{\mathbb{R}} \mathcal{U}^{h}(s, r, x) \tilde{N}(d r, d x), \quad s \in[t, T],
\end{aligned}
$$

where $m_{h}^{s, r}: \mathbb{R} \times \mathbb{R} \times \mathbb{R} \times \mathbb{R} \times \mathbb{R} \rightarrow \mathbb{R}$ is a linear function defined by $m_{h}^{s, r}(y, z, \zeta, u, \mu)=A_{h}^{2}(s, r) y+A_{h}^{3}(s, r) z+A_{h}^{4}(s, r) \zeta+A_{h}^{5}(s, r) u+A_{h}^{6}(s, r) \mu$, for

$$
\begin{array}{lll}
A_{h}^{1}(s, r)=\int_{0}^{1} D^{1} f\left(l_{h}^{s, r}(\theta) ;[t, T]\right) d \theta, & A_{h}^{2}(s, r)=\int_{0}^{1} \frac{\partial}{\partial y} f\left(l_{h}^{s, r}(\theta)\right) d \theta, \\
A_{h}^{3}(s, r)=\int_{0}^{1} \frac{\partial}{\partial z} f\left(l_{h}^{s, r}(\theta)\right) d \theta, & A_{h}^{4}(s, r)=\int_{0}^{1} \frac{\partial}{\partial \zeta} f\left(l_{h}^{s, r}(\theta)\right) d \theta, \\
A_{h}^{5}(s, r)=\int_{0}^{1} \frac{\partial}{\partial u} f\left(l_{h}^{s, r}(\theta)\right) d \theta, & A_{h}^{6}(s, r)=\int_{0}^{1} \frac{\partial}{\partial \mu} f\left(l_{h}^{s, r}(\theta)\right) d \theta .
\end{array}
$$


Additionally, we get with the inequality (28)

$$
\begin{aligned}
\mathbb{E}\left[\sup _{s \in[t, T]}\left|\mathcal{Y}^{h}(s)-\mathcal{Y}^{\bar{h}}(s)\right|^{2}\right] & \leq C\left(\mathbb{E}\left[\int_{t}^{T}\left|\Delta \Phi^{h}\left(X_{\gamma_{t}, s-}\right)-\Delta \Phi^{\bar{h}}\left(X_{\gamma_{t}, s-}\right)\right|^{2} d s\right]\right. \\
& \left.+\mathbb{E}\left[\int_{t}^{T} \int_{t}^{T}\left|A_{h}^{1}(s, r)-A_{\bar{h}}^{1}(s, r)\right|^{2} d r d s\right]\right) .
\end{aligned}
$$

We obtain for $h, \bar{h} \in \mathbb{R} \backslash\{0\}$ with (D1)

$$
\begin{aligned}
& \lim _{h \rightarrow \bar{h}} \mathbb{E}\left[\int_{t}^{T}\left|\Delta \Phi^{h}\left(X_{\gamma_{t}, s-}\right)-\Delta \Phi^{\bar{h}}\left(X_{\gamma_{t}, s-}\right)\right|^{2} d s\right] \\
& \leq \lim _{h \rightarrow \bar{h}} \mathbb{E}\left[\int_{t}^{T}\left|\frac{1}{\bar{h}} \Phi\left(X_{\gamma_{t}, s_{-}}\right)-\frac{1}{h} \Phi\left(X_{\gamma_{t}, s-}\right)\right|^{2} d s\right]=0 .
\end{aligned}
$$

(D4) provides

$$
\begin{aligned}
& \lim _{h \rightarrow \bar{h}} \mathbb{E}\left[\int_{t}^{T} \int_{t}^{T}\left|A_{h}^{1}(s, r)-A_{\bar{h}}^{1}(s, r)\right|^{2} d r d s\right] \\
& \leq \lim _{h \rightarrow \bar{h}} \mathbb{E}\left[\int_{t}^{T} \int_{t}^{T} \int_{0}^{1}\left|D^{1} f\left(l_{h}^{s, r}(\theta) ;[t, T]\right)-D^{1} f\left(l_{\bar{h}}^{s, r}(\theta) ;[t, T]\right)\right|^{2} d \theta d r d s\right]=0 .
\end{aligned}
$$

Thus, it follows

$$
\lim _{h \rightarrow \bar{h}} \mathbb{E}\left[\sup _{s \in[t, T]}\left|\mathcal{Y}^{h}(s)-\mathcal{Y}^{\bar{h}}(s)\right|^{2}\right]=0 .
$$

With the same arguments, we get

$$
\lim _{h \rightarrow \bar{h}} \mathbb{E}\left[\int_{t}^{T} \int_{t}^{T}\left|\mathcal{Z}^{h}(s, r)-\mathcal{Z}^{\bar{h}}(s, r)\right|^{2} d r d s\right]=0
$$

and

$$
\lim _{h \rightarrow \bar{h}} \mathbb{E}\left[\int_{t}^{T} \int_{t}^{T} \int_{\mathbb{R}}\left|\mathcal{U}^{h}(s, r, x)-\mathcal{U}^{\bar{h}}(s, r, x)\right|^{2} v(d x) d r d s\right]=0 .
$$

Finally, this provides

$$
\lim _{h \rightarrow \bar{h}}\left\|\left(\mathcal{Y}^{h}, \mathcal{Z}^{h}, \mathcal{U}^{h}\right)-\left(\mathcal{Y}^{\bar{h}}, \mathcal{Z}^{\bar{h}}, \mathcal{U}^{\bar{h}}\right)\right\|_{\mathcal{H}^{2}[t, T]}^{2}=0 .
$$

Since $S^{2}[t, T], L^{2}\left(t, T ; M^{2}[t, T]\right)$ and $L^{2}\left(t, T ; L_{N}^{2}(t, T)\right)$ are Banach spaces, the sequence $\mathcal{Y}^{h_{n}}(s)$ converges to a process $D^{1} Y_{\gamma_{t}}(s ;[t, T])$, the sequence $\mathcal{Z}^{h_{n}}(s, r)$ converges to a process $D^{1} Z_{\gamma_{t}}(s, r ;[t, T])$, and the process $\mathcal{U}^{h_{n}}(s, r, x)$ converges to $D^{1} U_{\gamma_{t}}(s, r, x ;[t, T])$ for a sequence $\left(h_{n}\right)_{n} \in \mathbb{R} \backslash\{0\}$ and with respect to the corresponding norms.

This implies that $\left(D^{1} Y_{\gamma_{t}}(\cdot ;[t, T]), D^{1} Z_{\gamma_{t}}(\cdot, \cdot ;[t, T]), D^{1} U_{\gamma_{t}}(\cdot, \cdot, \cdot ;[t, T])\right) \in$ $\mathcal{H}^{2}[t, T]$ is the adapted $M$-solution to the path-dependent BSVIE with jumps (29). 


\section{Duality Principle and Comparison Theorem}

In this section, we prove the duality principle for linear path-dependent BSVIEs with jumps

$$
\begin{aligned}
Y_{\gamma_{t}}(s)= & \Phi\left(X_{\gamma_{t}, s}\right)+\int_{s}^{T}\left[A^{T}\left(s, r, X_{\gamma_{t}, r-}\right) Y_{\gamma_{t}}(r-)+\sum_{j=1}^{d} B_{j}^{T}\left(s, X_{\gamma_{t}, s-}\right) Z_{\gamma_{t}}^{j}(r, s)\right. \\
& \left.+\int_{\mathbb{R}^{l}} C^{T}\left(s, X_{\gamma_{t}, s-}, z\right) U_{\gamma_{t}}(r, s, z) v(d z)\right] d r \\
& -\int_{s}^{T} Z_{\gamma_{t}}(s, r) d W(r)-\int_{s}^{T} \int_{\mathbb{R}^{l}} U_{\gamma_{t}}(s, r, z) \tilde{N}(d r, d z), \quad s \in[t, T],
\end{aligned}
$$

and linear path-dependent FSVIEs with jumps of the form

$$
\begin{aligned}
X_{\gamma_{t}}(s)= & \varphi\left(X_{\gamma_{t}, s}\right)+\int_{t}^{s} A\left(r, s, X_{\gamma_{t}, s-}\right) X_{\gamma_{t}}(r-) d r+\sum_{j=1}^{d} \int_{t}^{s} B_{j}\left(r, X_{\gamma_{t}, r-}\right) X_{\gamma_{t}}(r-) d W_{j}(r) \\
& +\int_{t}^{s} \int_{\mathbb{R}^{l}} C\left(r, X_{\gamma_{t}, r-}, z\right) X_{\gamma_{t}}(r-) \tilde{N}(d r, d z), \quad s \in[t, T], \\
X_{\gamma_{t}}(s)= & \gamma_{t}(s), \quad s \in[0, t),
\end{aligned}
$$

where it is necessary that $m=d$ for the dimensions. First, we show that such a FSVIE admits an adapted solution. We consider a more general path-dependent FSVIE with jumps of the form

$$
\begin{aligned}
X_{\gamma_{t}}(s)= & \varphi\left(X_{\gamma_{t}, s}\right)+\int_{t}^{s} \bar{A}\left(r, s, X_{\gamma_{t}, s-}\right) d r+\sum_{j=1}^{d} \int_{t}^{s} \bar{B}_{j}\left(r, X_{\gamma_{t}, r-}\right) d W_{j}(r) \\
& +\int_{t}^{s} \int_{\mathbb{R}^{l}} \bar{C}\left(r, X_{\gamma_{t}, r-}, z\right) \tilde{N}(d r, d z), \quad s \in[t, T], \\
X_{\gamma_{t}}(s)= & \gamma_{t}(s), \quad s \in[0, t),
\end{aligned}
$$

and assume the following.

(C1) Let $\varphi(\cdot) \in L_{\mathbb{F}}^{2}\left(\Omega \times D\left([0, T], \mathbb{R}^{d}\right) ; \mathbb{R}^{d}\right)$ with

$$
|\varphi(\gamma)|^{2} \leq K\left(1+\sup _{u \in[0, T]}|\gamma(u)|^{2}\right)
$$

and

$$
\left|\varphi\left(\gamma_{s}\right)-\varphi\left(\bar{\gamma}_{\bar{s}}\right)\right|^{2} \leq K\left(\sup _{u \in[0, T]}|\gamma(u)-\bar{\gamma}(u)|^{2}+|s-\bar{s}|^{2}\right),
$$

for any $\gamma, \bar{\gamma} \in D\left([0, T], \mathbb{R}^{d}\right)$ and $s, \bar{s} \in[t, T]$.

(C2) The process $\bar{A}: \Omega \times \Delta^{c}[t, T] \times D\left([0, T], \mathbb{R}^{d}\right) \rightarrow \mathbb{R}^{d}$ is $\mathcal{F}_{T}^{t} \otimes \mathcal{B}\left(\Delta^{c}[t, T] \times\right.$ $\left.D\left([0, T], \mathbb{R}^{d}\right)\right)$-measurable such that $r \mapsto \bar{A}(r, s, \gamma)$ is progressively measurable 
on $[t, s]$ for any $s \in[t, T], \gamma \in D\left([0, T], \mathbb{R}^{d}\right)$. Moreover, map $\bar{A}$ is Lipschitz continuous in the sense that

$$
|\bar{A}(r, s, \gamma)-\bar{A}(r, \bar{s}, \bar{\gamma})|^{2} \leq K\left(\sup _{\tau \in[t, r]}|\gamma(\tau)-\bar{\gamma}(\tau)|^{2}+|s-\bar{s}|^{2}\right),
$$

a.e. $(\omega, r) \in \Omega \times[t, s \wedge \bar{s}]$ for all $s, \bar{s} \in[t, T], \gamma, \bar{\gamma} \in D\left([0, T], \mathbb{R}^{d}\right)$, and satisfies the following growth condition

$$
|\bar{A}(r, s, \gamma)|^{2} \leq K\left(1+\sup _{u \in[0, s]}|\gamma(u)|^{2}\right) .
$$

(C3) The map $(r, x, z) \mapsto\left(\bar{B}_{1}(r, x), \ldots, \bar{B}_{d}(r, x), \bar{C}(r, x, z)\right)$ defined on $[t, T] \times$ $D\left([0, T], \mathbb{R}^{d}\right) \times \mathbb{R}^{l}$ is Borel measurable and the following Lipschitz and growth conditions hold

$\sum_{j=1}^{d}\left|\bar{B}_{j}(s, \gamma)-\bar{B}_{j}(s, \bar{\gamma})\right|^{2}+\int_{\mathbb{R}^{l}}|\bar{C}(s, \gamma, z)-\bar{C}(s, \bar{\gamma}, z)|^{2} v(d z) \leq K \sup _{u \in[0, s]}|\gamma(u)-\bar{\gamma}(u)|^{2}$

and

$$
\sum_{j=1}^{d}\left|\bar{B}_{j}(s, \gamma)\right|^{2}+\int_{\mathbb{R}^{l}}|\bar{C}(s, \gamma, z)|^{2} v(d z) \leq K\left(1+\sup _{u \in[0, s]}|\gamma(u)|^{2}\right),
$$

for each $s \in[t, T]$ and for all $\gamma, \bar{\gamma} \in D\left([0, T], \mathbb{R}^{d}\right)$.

Theorem 4 Let (C1)-(C3) hold. Then, for any $\gamma \in D\left([0, T], \mathbb{R}^{d}\right)$ with $\sup _{s \in[0, t]}|\gamma(s)|^{2}<\infty, t \in[0, T]$, there exists a strong solution to FSVIE (32).

Proof The proof is quite similar to Kromer et al. (2015).

Now, let $m=d$ and we consider the linear cases of path-dependent FSVIEs and BSVIEs with jumps (31) and (30), where we regard

$$
\begin{aligned}
\bar{A}(r, s, \gamma) & =A(r, s, \gamma) \gamma(r-), \\
\bar{B}_{j}(r, \gamma) & =B_{j}(r, \gamma) \gamma(r-), \quad j=1, \cdots, d, \\
\bar{C}(r, \gamma, z) & =C(r, \gamma, z) \gamma(r-),
\end{aligned}
$$

for any $(r, s, \gamma) \in[t, T]^{2} \times \mathbb{R}^{l}$ and with $A(\cdot, \cdot, \cdot), B_{j}(\cdot, \cdot), C(\cdot, \cdot) \in \mathbb{R}^{d \times d}$.

Theorem 5 Let $\Phi \in L_{\mathcal{F}_{T}^{t}}^{2}\left(D\left([0, T], \mathbb{R}^{d}\right) ; \mathbb{R}\right), \quad \gamma \in D\left([0, T], \mathbb{R}^{d}\right)$, with $\sup _{s \in[0, t]}|\gamma(s)|^{2}<\infty, t \in[0, T]$ and $\varphi(\cdot) \in L_{\mathbb{F}}^{2}\left(\Omega \times D\left([0, T], \mathbb{R}^{d}\right) ; \mathbb{R}^{d}\right)$ satisfying (C1).

Furthermore, $A: \Omega \times[t, T]^{2} \times D\left([0, T], \mathbb{R}^{d}\right) \rightarrow \mathbb{R}^{d \times d}, B_{j}: \Omega \times[t, T] \times$ $D\left([0, T], \mathbb{R}^{d}\right) \rightarrow \mathbb{R}^{d \times d}, C: \Omega \times[t, T] \times D\left([0, T], \mathbb{R}^{d}\right) \times \mathbb{R}^{l} \rightarrow \mathbb{R}^{d \times d}$ are uniformly bounded and (C2)-(C3) hold for (33)-(35).

Let $X_{\gamma_{t}}$ be the strong solution to FSVIE (31) and $\left(Y_{\gamma_{t}}(\cdot), Z_{\gamma_{t}}(\cdot, \cdot), U_{\gamma_{t}}(\cdot, \cdot, \cdot)\right) \in$ $\mathcal{H}^{2}[t, T]$ be the unique adapted M-solution to BSVIE (30). Then, we have the 
following duality principle

$$
\mathbb{E}\left[\int_{t}^{T}\left\langle\varphi\left(X_{\gamma_{t}, r-}\right), Y_{\gamma_{t}}(r-)\right\rangle d r\right]=\mathbb{E}\left[\int_{t}^{T}\left\langle X_{\gamma_{t}}(r-), \Phi\left(X_{\gamma_{t}, r-}\right)\right\rangle d r\right] .
$$

Proof Since $\left(Y_{\gamma_{t}}(\cdot), Z_{\gamma_{t}}(\cdot, \cdot), U_{\gamma_{t}}(\cdot, \cdot, \cdot)\right) \in \mathcal{H}^{2}[t, T]$ is the unique adapted Msolution to BSVIE (30), we get

$$
Y_{\gamma_{t}}(s)=\mathbb{E}\left[Y_{\gamma_{t}}(s)\right]+\int_{t}^{s} Z_{\gamma_{t}}(s, r) d W(r)+\int_{t}^{s} \int_{\mathbb{R}^{l}} U_{\gamma_{t}}(s, r, z) \tilde{N}(d r, d z) .
$$

With Fubini's theorem, this implies

$$
\begin{aligned}
\mathbb{E} & {\left[\int_{t}^{T}\left\langle\varphi\left(X_{\gamma_{t}, r-}\right), Y_{\gamma_{t}}(r-)\right\rangle d r\right] } \\
= & \mathbb{E}\left[\int_{t}^{T}\left\langle X_{\gamma_{t}}(s-), Y_{\gamma_{t}}(s-)\right\rangle d s\right]-\mathbb{E}\left[\int_{t}^{T}\left\langle\int_{t}^{s} A\left(r, s, X_{\gamma_{t}, s-}\right) X_{\gamma_{t}}(r-) d r, Y_{\gamma_{t}}(s-)\right\rangle d s\right] \\
& -\mathbb{E}\left[\int _ { t } ^ { T } \left\langle\int_{t}^{s} \sum_{j=1}^{d} B_{j}\left(r, s, X_{\gamma_{t}, s-}\right) X_{\gamma_{t}}(r-) d W_{j}(r)\right.\right. \\
& +\int_{t}^{s} \int_{\mathbb{R}^{l}} C\left(r, s, X_{\gamma_{t}, s-}, z\right) X_{\gamma_{t}}(r-) \tilde{N}(d r, d z), \\
& \left.\left.\mathbb{E}\left[Y_{\gamma_{t}}(s-)\right]+\int_{t}^{s} Z_{\gamma_{t}}(s, r) d W(r)+\int_{t}^{s} \int_{\mathbb{R}^{l}} U_{\gamma_{t}}(s, r, z) \tilde{N}(d r, d z)\right\rangle d s\right] \\
= & {\left[\int _ { t } ^ { T } \left\langleX_{\gamma_{t}}(s-), Y_{\gamma_{t}}(s-)-\int_{s}^{T}\left(A^{T}\left(s, r, X_{\gamma_{t}, r-}\right) Y_{\gamma_{t}}(r-)+\sum_{j=1}^{d} B_{j}^{T}\left(s, r, X_{\gamma_{t}, r-}\right) Z_{\gamma_{t}}^{j}(r, s)\right.\right.\right.} \\
& \left.\left.\left.\left.+\int_{\mathbb{R}^{l}} C^{T}\left(s, r, X_{\gamma_{t}, r-}, z\right) U_{\gamma_{t}}(r, s, z)\right\rangle \nu(d z)\right) d r\right\rangle d s\right] \\
= & \mathbb{E}\left[\int_{t}^{T}\left\langle X_{\gamma_{t}}(s-), \Phi\left(X_{\gamma_{t}, s-}\right)\right\rangle d s\right] .
\end{aligned}
$$

Next, we consider a one-dimensional linear path-dependent FSVIE with jumps of the form

$$
\begin{aligned}
X_{\gamma_{t}}(s)= & \varphi\left(X_{\gamma_{t}, s}\right)+\int_{t}^{s} a\left(r, s, X_{\gamma_{t}, s-}\right) X_{\gamma_{t}}(r-) d r+\int_{t}^{s} b(r) X_{\gamma_{t}}(r-) d W(r) \\
& +\int_{t}^{s} \int_{\mathbb{R}^{l}} c(r, z) X_{\gamma_{t}}(r-) \tilde{N}(d r, d z), \quad s \in[t, T], \\
X_{\gamma_{t}}(s)= & \gamma_{t}(s), \quad s \in[0, t),
\end{aligned}
$$

where the coefficients $b$ and $c$ do not depend on the path of process $X_{\gamma_{t}}$. For the proof of the comparison theorem to BSVIEs we need a comparison theorem for FSVIEs. 
Theorem 6 Let $a: \Omega \times[t, T]^{2} \times D([0, T], \mathbb{R}) \rightarrow \mathbb{R}$ be $\mathcal{F}_{T}^{t} \otimes \mathcal{B}\left([t, T]^{2} \times\right.$ $D([0, T], \mathbb{R}))$-measurable such that for any $s \in[t, T], \gamma \in D([0, T], \mathbb{R})$ the map $r \rightarrow a\left(r, s, \gamma_{s-}\right)$ is $\mathbb{F}$-progressively measurable on $[t, s]$. Moreover, a satisfies the following Lipschitz and growth conditions

$$
\left|a\left(r, s, \gamma_{s-}\right)-a\left(r, \bar{s}, \bar{\gamma}_{\bar{s}-}\right)\right|^{2} \leq K\left(\sup _{s \in[t, r]}|\gamma(s)-\bar{\gamma}(s)|^{2}+|s-\bar{s}|^{2}\right),
$$

a.e. $(\omega, r) \in \Omega \times[t, s \wedge \bar{s}]$ for all $s, \bar{s} \in[t, T], \gamma, \bar{\gamma} \in D([0, T], \mathbb{R})$, and

$$
\left|a\left(r, s, \gamma_{s-}\right)\right|^{2} \leq K\left(1+\sup _{s \in[t, r]}|\gamma(s)|^{2}\right),
$$

a.e. $(\omega, r) \in \Omega \times[t, s]$ for all $s \in[t, T], \gamma \in D([0, T], \mathbb{R})$. Furthermore, let

$$
a\left(r, \bar{s}, \gamma_{\bar{s}-}\right)-a\left(r, s, \gamma_{s-}\right) \geq 0,
$$

a.e. $(\omega, r) \in \Omega \times[t, s]$ for any $\gamma \in D([0, T], \mathbb{R}), s, \bar{s} \in[t, T]$ with $\bar{s} \geq s$. Let $(r, z) \rightarrow(b(r), c(r, z))$ be Borel measurable, where $b$ is uniformly bounded and

$$
\int_{\mathbb{R}^{l}}|c(r, z)|^{2} v(d z)<\infty
$$

for any $r \in[t, T]$. In addition, $c(r, z)>-1$ a.s. for all $(r, z) \in[t, T] \times \mathbb{R}^{l}$. Then, for any $\varphi(\cdot) \in L_{\mathbb{F}}^{2}(\Omega \times D([0, T], \mathbb{R}) ; \mathbb{R})$, satisfying $(C 1)$, with

$$
\varphi\left(\gamma_{\bar{s}}\right) \geq \varphi\left(\gamma_{s}\right) \geq 0, \quad s, \bar{s} \in[t, T], \bar{s} \geq s, \quad \text { a.s. },
$$

FSVIE (37) admits a unique solution $X_{\gamma_{t}}(\cdot)$. Moreover, it is

$$
X_{\gamma_{t}}(s) \geq 0, \quad s \in[t, T], \text { a.s. }
$$

Proof We will use the techniques from Wang and Yong (2015) and Lu (2016). Let $\Pi=\left\{t_{k}, 0 \leq k \leq N\right\}$ be a finite sequence of real numbers with $t=t_{0}<t_{1}<\ldots<$ $t_{N}=T,\|\Pi\|=\max _{1 \leq k \leq N}\left|t_{k}-t_{k-1}\right|$.

Define

$$
\begin{aligned}
a^{\Pi}\left(r, s, \gamma_{s-}\right) & =\sum_{k=0}^{N-1} a\left(r, t_{k}, \gamma_{t_{k}}\right) \mathbb{1}_{\left[t_{k}, t_{k+1}\right)}(s), \\
\varphi^{\Pi}\left(\gamma_{s-}\right) & =\sum_{k=0}^{N-1} \varphi\left(\gamma_{t_{k}}\right) \mathbb{1}_{\left[t_{k}, t_{k+1}\right)}(s),
\end{aligned}
$$

where $\varphi\left(X_{\gamma_{t}, t_{k}}\right) \leq \varphi\left(X_{\gamma_{t}, t_{k+1}}\right)$ a.s.

For any $t \leq r \leq s \leq T, \gamma \in D\left([0, T], \mathbb{R}^{d}\right)$, we note that with (C2)

$$
\left|a^{\Pi}\left(r, s, X_{\gamma_{t}, s-}\right)-a\left(r, s, X_{\gamma_{t}, s-}\right)\right| \leq K\left(\sup _{\tau \in[s, \bar{s}],|s-\bar{s}| \leq\|\Pi\|}\left|X_{\gamma_{t}, s}(\tau)-X_{\gamma_{t}, \bar{s}}(\tau)\right|+\|\Pi\|\right) \text {. }
$$

Furthermore, we obtain

$\mathbb{E}\left[\sup _{s \in[t, T]}\left|\varphi^{\Pi}\left(X_{\gamma_{t}, s}\right)-\varphi\left(X_{\gamma_{t}, s}\right)\right|^{2}\right] \leq \mathbb{E}\left[\sup _{|s-\bar{s}| \leq\|\Pi\|, s, \bar{s} \in[t, T]}\left|\varphi\left(X_{\gamma_{t}, s}\right)-\varphi\left(X_{\gamma_{t}, \bar{s}}\right)\right|^{2}\right]$. 
Now, let $X^{\Pi}(\cdot)$ be the solution to the following FSVIE with jumps

$$
\begin{aligned}
X_{\gamma_{t}}^{\Pi}(s)= & \varphi^{\Pi}\left(X_{\gamma_{t}, s}\right)+\int_{t}^{s} a^{\Pi}\left(r, s, X_{\gamma_{t}, s-}\right) X_{\gamma_{t}}^{\Pi}(r-) d r+\int_{t}^{s} b(r) X_{\gamma_{t}}^{\Pi}(r-) d W(r) \\
& +\int_{t}^{s} \int_{\mathbb{R}^{l}} c(r, z) X_{\gamma_{t}}^{\Pi}(r-) \tilde{N}(d r, d z), s \in[t, T], \\
X_{\gamma_{t}}^{\Pi}(s)= & \gamma(s), \quad s \in[0, t) .
\end{aligned}
$$

We get

$$
\begin{aligned}
& \mathbb{E}\left[\sup _{s \in[t, T]}\left|X_{\gamma_{t}}^{\Pi}(s)-X_{\gamma_{t}}(s)\right|^{2}\right] \\
& \leq C \mathbb{E}\left[\sup _{s \in[t, T]}\left|\varphi^{\Pi}\left(X_{\gamma_{t}, s}\right)-\varphi\left(X_{\gamma_{t}, s}\right)\right|^{2}+\sup _{s \in[t, T]}\left|\int_{t}^{s} b(r)\left(X_{\gamma_{t}}^{\Pi}(r-)-X_{\gamma_{t}}(r-)\right) d W(r)\right|^{2}\right. \\
& \quad+\sup _{s \in[t, T]}\left(\int_{t}^{s}\left|a^{\Pi}\left(r, s, X_{\gamma_{t}, s-}\right) X_{\gamma_{t}}^{\Pi}(r-)-a\left(r, s, X_{\gamma_{t}, s-}\right) X_{\gamma_{t}}(r-)\right| d r\right)^{2} \\
& \left.\quad+\sup _{s \in[t, T]}\left|\int_{t}^{s} \int_{\mathbb{R}^{l}} c(r, z)\left(X_{\gamma_{t}}^{\Pi}(r-)-X_{\gamma_{t}}(r-)\right) \tilde{N}(d r, d z)\right|^{2}\right] .
\end{aligned}
$$

It is

$$
\begin{aligned}
& \left|a^{\Pi}\left(r, s, X_{\gamma_{t}, s-}\right) X_{\gamma_{t}}^{\Pi}(r-)-a\left(r, s, X_{\gamma_{t}, s-}\right) X_{\gamma_{t}}(r-)\right| \\
& \leq\left|a^{\Pi}\left(r, s, X_{\gamma_{t}, s-}\right)\right|\left|X_{\gamma_{t}}^{\Pi}(r-)-X_{\gamma_{t}}(r-)\right|+\left|a^{\Pi}\left(r, s, X_{\gamma_{t}, s-}\right)-a\left(r, s, X_{\gamma_{t}, s-}\right)\right|\left|X_{\gamma_{t}}(r-)\right|
\end{aligned}
$$

and

$$
\begin{aligned}
& \mathbb{E}\left[\sup _{s \in[t, T]}\left(\int_{t}^{s}\left|a^{\Pi}\left(r, s, X_{\gamma_{t}, s-}\right)\right|\left|X_{\gamma_{t}}^{\Pi}(r-)-X_{\gamma_{t}}(r-)\right| d r\right)^{2}\right] \\
& \leq \mathbb{E}\left[\sup _{s \in[t, T]} \int_{t}^{s} K \cdot\left(1+\sup _{\tau \in[t, r]}\left|X_{\gamma_{t}}(\tau)\right|^{2}\right)\left|X_{\gamma_{t}}^{\Pi}(r-)-X_{\gamma_{t}}(r-)\right|^{2} d r\right] \\
& \leq K \mathbb{E}\left[\sup _{\tau \in[s, \bar{s}], s, \bar{s} \leq\|\Pi\|}\left|X_{\gamma_{t}}^{\Pi}(r-)-X_{\gamma_{t}}(r-)\right|^{2} \int_{t}^{T} \sup _{\tau \in[t, r]}\left|X_{\gamma_{t}}(\tau)\right|^{2} d r\right. \\
& \left.\quad+\int_{t}^{T}\left|X_{\gamma_{t}}^{\Pi}(r-)-X_{\gamma_{t}}(r-)\right|^{2} d r\right] .
\end{aligned}
$$

Since $b: \omega \times[t, T] \rightarrow \mathbb{R}, c: \omega \times[t, T] \times \mathbb{R}^{l} \rightarrow \mathbb{R}$ are Borel measurable and $X_{\gamma_{t}}^{\Pi}, X_{\gamma_{t}}$ are both càdlàg and adapted processes, which implies that $X_{\gamma_{t}}^{\Pi}$ and $X_{\gamma_{t}}$ are progressively measurable, the integrals

$\int_{t} b(r)\left(X_{\gamma_{t}}^{\Pi}(r-)-X_{\gamma_{t}}(r-)\right) d W(r)$ and $\int_{t} \int_{\mathbb{R}^{l}} c(r, z)\left(X_{\gamma_{t}}^{\Pi}(r-)-X_{\gamma_{t}}(r-)\right) \tilde{N}(d r, d z)$ 
are local martingales. Since $b: \omega \times[t, T] \rightarrow \mathbb{R}$ is bounded, we get with BurkholderDavis- Gundy inequality

$$
\begin{aligned}
& \mathbb{E}\left[\sup _{s \in[t, T]}\left|\int_{t}^{s} b(r)\left(X_{\gamma_{t}}^{\Pi}(r-)-X_{\gamma_{t}}(r-)\right) d W(r)\right|^{2}\right] \\
& \leq C \mathbb{E}\left[\int_{t}^{T}\left|X_{\gamma_{t}}^{\Pi}(r-)-X_{\gamma_{t}}(r-)\right|^{2} d r\right] .
\end{aligned}
$$

Similarly, it is

$$
\begin{aligned}
& \mathbb{E}\left[\left|\int_{t}^{s} \int_{\mathbb{R}^{l}} c(r, z)\left(X_{\gamma_{t}}^{\Pi}(r-)-X_{\gamma_{t}}(r-)\right) \tilde{N}(d r, d z)\right|^{2}\right] \\
& \leq C \mathbb{E}\left[\int_{t}^{T}\left|X_{\gamma_{t}}^{\Pi}(r-)-X_{\gamma_{t}}(r-)\right|^{2} d r\right]
\end{aligned}
$$

and with Gronwall's lemma we get for any $s \in[t, T]$

$$
\begin{aligned}
\mathbb{E} & {\left[\left|X_{\gamma_{t}}^{\Pi}(s)-X_{\gamma_{t}}(s)\right|^{2}\right] } \\
\leq & C\left\{\mathbb{E}\left[\sup _{s, \bar{s} \in[t, T],|s-\bar{s}| \leq\|\Pi\|}\left|\varphi\left(X_{\gamma_{t}, s}\right)-\varphi\left(X_{\gamma_{t}, \bar{s}}\right)\right|^{2}\right]\right. \\
& \left.+\mathbb{E}\left[\left(\sup _{\tau \in[s, \bar{s}],|s-\bar{s}| \leq\|\Pi\|}\left|X_{\gamma_{t}, s-}(\tau)-X_{\gamma_{t}, \bar{s}-}(\tau)\right|^{2}+\|\Pi\|^{2}\right) \int_{t}^{T}\left|X_{\gamma_{t}}(r-)\right|^{2} d r\right]\right\} e^{C(T-t)} .
\end{aligned}
$$

Hence, it follows for any $s \in[t, T]$

$$
\lim _{\|\Pi\| \rightarrow 0} \mathbb{E}\left[\left|X_{\gamma_{t}}^{\Pi}(s)-X_{\gamma_{t}}(s)\right|^{2}\right]=0 .
$$

For $s \in\left[t, t_{1}\right)$ we get with definition (43) a linear FSDE of the form

$$
\begin{aligned}
X_{\gamma_{t}}^{\Pi}(s)= & \varphi\left(X_{\gamma_{t}, t}\right)+\int_{t}^{s} a\left(r, t, X_{\gamma_{t}, t}\right) X_{\gamma_{t}}^{\Pi}(r-) d r+\int_{t}^{s} b(r) X_{\gamma_{t}}^{\Pi}(r-) d W(r) \\
& +\int_{t}^{s} \int_{\mathbb{R}} c(r, z) X_{\gamma_{t}}^{\Pi}(r-) \tilde{N}(d r, d z)
\end{aligned}
$$

where the solution $X_{\gamma_{t}}^{\Pi}(s), s \in\left[t, t_{1}\right)$, is a Doléans-Dade exponential of the semimartingale $X_{\gamma_{t}}$ which is positive for $c(r, z)>-1$ for all $(s, z) \in\left[t, t_{1}\right) \times \mathbb{R}^{l}$, see Doléans-Dade (1970). In particular, it is

$$
\begin{aligned}
X_{\gamma_{t}}^{\Pi}\left(t_{1}-\right)= & \varphi\left(X_{\gamma_{t}, t}\right)+\int_{t}^{t_{1}} a\left(r, t, X_{\gamma_{t}, t}\right) X_{\gamma_{t}}^{\Pi}(r-) d r+\int_{t}^{t_{1}} b(r) X_{\gamma_{t}}^{\Pi}(r-) d W(r) \\
& +\int_{t}^{t_{1}} \int_{\mathbb{R}} c(r, z) X_{\gamma_{t}}^{\Pi}(r-) \tilde{N}(d r, d z) .
\end{aligned}
$$


Now, for $s \in\left[t_{1}, t_{2}\right)$ it is

$$
\begin{aligned}
X_{\gamma_{t}}^{\Pi}(s)= & \hat{\varphi}\left(X_{\gamma_{t}, t_{1}}\right)+\int_{t_{1}}^{s} a\left(r, t_{1}, X_{\gamma_{t}, t_{1}}\right) X_{\gamma_{t}}^{\Pi}(r-) d r+\int_{t_{1}}^{s} b(r) X_{\gamma_{t}}^{\Pi}(r-) d W(r) \\
& +\int_{t_{1}}^{s} \int_{\mathbb{R}} c(r, z) X_{\gamma_{t}}^{\Pi}(r-) \tilde{N}(d r, d z),
\end{aligned}
$$

where

$\hat{\varphi}\left(X_{\gamma_{t}, t_{1}}\right)=\varphi\left(X_{\gamma_{t}, t_{1}}\right)-\varphi\left(X_{\gamma_{t}, t}\right)+X_{\gamma_{t}}^{\Pi}\left(t_{1}-\right)+\int_{t}^{t_{1}}\left(a\left(r, t_{1}, X_{\gamma_{t}, t_{1}}\right)-a\left(r, t, X_{\gamma_{t}, t}\right)\right) X_{\gamma_{t}}^{\Pi}(r-) d r$.

Since (40) holds, $\varphi\left(X_{\gamma_{t}, t_{1}}\right) \geq \varphi\left(X_{\gamma_{t}, t}\right)$ for $t \leq t_{1}$ and $X_{\gamma_{t}}^{\Pi}\left(t_{1}-\right) \geq 0$, it is $\hat{\varphi}\left(X_{\gamma_{t}, t_{1}}\right) \geq 0$. With the same arguments for $s \in\left[t, t_{1}\right)$, it is $X_{\gamma_{t}}^{\Pi}(s) \geq 0$ a.s. for any $s \in\left[t_{1}, t_{2}\right)$. By induction we get that $X_{\gamma_{t}}^{\Pi}(s) \geq 0$ a.s. for all $s \in[t, T]$. Equation (44) yields that $X_{\gamma_{t}}(s) \geq 0$ a.s. for any $s \in[t, T]$.

For the comparison theorem we consider the following path-dependent BSVIE with jumps

$$
\begin{aligned}
& Y_{\gamma_{t}}(s) \\
& =\Phi\left(X_{\gamma_{t}, s}\right)+\int_{s}^{T} f\left(s, r, X_{\gamma_{t}, r-}, Y_{\gamma_{t}}(r-)\right)+h\left(s, Z_{\gamma_{t}}(r, s), \int_{\mathbb{R}^{l}} U_{\gamma_{t}}(r, s, z) v(d z)\right) d r \\
& \quad-\int_{s}^{T} Z_{\gamma_{t}}(s, r) d W(r)-\int_{s}^{T} \int_{\mathbb{R}^{l}} U_{\gamma_{t}}(s, r, z) \tilde{N}(d r, d z), \quad s \in[t, T] .
\end{aligned}
$$

Theorem 7 Let $f, \bar{f}: \Omega \times[t, T]^{2} \times D\left([0, T], \mathbb{R}^{d}\right) \times \mathbb{R} \rightarrow \mathbb{R}$ and $h, \bar{h}: \Omega \times$ $[t, T] \times D\left([0, T], \mathbb{R}^{d}\right) \times \mathbb{R} \times \mathbb{R} \rightarrow \mathbb{R}$ satisfy assumptions (H2) and (H3) such that

$$
\begin{aligned}
f(s, r, \gamma, y) & \geq \bar{f}(s, r, \gamma, y,), \quad \text { a.s. }, \\
h(s, z, u) & \geq \bar{h}(s, z, u), \quad \text { a.s. }
\end{aligned}
$$

for all $(s, r, \gamma, y, z, u) \in \Delta^{c}[t, T] \times D\left([0, T], \mathbb{R}^{d}\right) \times \mathbb{R} \times \mathbb{R} \times \mathbb{R}$. Moreover, $y \mapsto$ $f(s, r, \gamma, y) \in C^{1}(\mathbb{R} ; \mathbb{R})$ and $(z, u) \mapsto h(s, z, u) \in C^{1}(\mathbb{R} \times \mathbb{R} ; \mathbb{R})$ for any $\gamma \in$ $D([0, T], \mathbb{R})$ and $(s, r) \in[t, T]^{2}$ with $f_{y}, h_{z}, h_{u}$ uniformly bounded and

$$
\left|f_{y}\left(r, s, \gamma_{s-}, y\right) \gamma(r-)-f_{y}\left(r, \bar{s}, \bar{\gamma}_{\bar{s}-}, y\right) \bar{\gamma}(r-)\right|^{2} \leq K\left(\sup _{s \in[t, r]}|\gamma(s)-\bar{\gamma}(s)|^{2}+|s-\bar{s}|^{2}\right) .
$$

Furthermore,

$$
f_{y}\left(r, \bar{s}, \gamma_{\bar{s}-}, y\right)-f_{y}\left(r, s, \gamma_{s-}, y\right) \geq 0,
$$

a.e. $(\omega, r) \in \Omega \times[t, s]$ for any $\gamma \in D([0, T], \mathbb{R}), s, \bar{s} \in[t, T]$ with $\bar{s} \geq s$. For $\Phi, \bar{\Phi} \in$ $L_{\mathcal{F}_{T}^{t}}^{2}\left(D\left([0, T], \mathbb{R}^{d}\right) ; \mathbb{R}\right)$ we assume $\Phi(\gamma) \geq \bar{\Phi}(\gamma)$ a.s. for all $\gamma \in D\left([0, T], \mathbb{R}^{d}\right)$.

Let $\left(Y_{\gamma_{t}}(\cdot), Z_{\gamma_{t}}(\cdot, \cdot), U_{\gamma_{t}}(\cdot, \cdot, \cdot)\right)$ and $\left(\bar{Y}_{\gamma_{t}}(\cdot), \bar{Z}_{\gamma_{t}}(\cdot, \cdot), \bar{U}_{\gamma_{t}}(\cdot, \cdot, \cdot)\right)$ be the adapted M-solution to BSVIE (45) corresponding to $(f, h, \Phi)$ and $(\bar{f}, \bar{h}, \bar{\Phi})$, respectively. 
Then, we get

$$
\mathbb{E}\left[\int_{t}^{T} Y_{\gamma_{t}}(u-) d u\right] \geq \mathbb{E}\left[\int_{t}^{T} \bar{Y}_{\gamma_{t}}(u-) d u\right]
$$

Proof Define for any $(s, r) \in[t, T]^{2}, h \in \mathbb{R} \backslash\{0\}$, the map $l_{X_{\gamma_{t}, r-}}^{s, r}:[0,1] \rightarrow \mathbb{R}$ by

$$
l_{X_{\gamma_{t}, r-}}^{s, r}(\theta)=\bar{Y}_{\gamma_{t}}(r-)+\theta\left(Y_{\gamma_{t}}(r-)-\bar{Y}_{\gamma_{t}}(r-)\right)
$$

It follows that

$$
\begin{aligned}
& f\left(s, r, X_{\gamma_{t}, r-}, Y_{\gamma_{t}}(r-)\right)-f\left(s, r, X_{\gamma_{t}, r-}, \bar{Y}_{\gamma_{t}}(r-)\right) \\
& =\int_{0}^{1} \frac{\partial}{\partial y} f\left(l_{X_{\gamma_{t}, r-}}^{s, r}(\theta)\right) d \theta \cdot\left(Y_{\gamma_{t}}(r-)-\bar{Y}_{\gamma_{t}}(r-)\right) .
\end{aligned}
$$

Similarly, we define for any $s \in[t, T], h \in \mathbb{R} \backslash\{0\}$, a map $g^{s}:[0,1] \rightarrow \mathbb{R} \times \mathbb{R}$ by

$$
\begin{aligned}
g^{s}(\theta)= & \left(\bar{Z}_{\gamma_{t}}(r, s)+\theta\left(Z_{\gamma_{t}}(r, s)-\bar{Z}_{\gamma_{t}}(r, s)\right),\right. \\
& \left.\int_{\mathbb{R}} \bar{U}_{\gamma_{t}}(r, s, z)+\theta\left(U_{\gamma_{t}}(r, s, z)-\bar{U}_{\gamma_{t}}(r, s, z)\right) v(d z)\right)
\end{aligned}
$$

and this yields

$$
\begin{aligned}
& h\left(s, Z_{\gamma_{t}}(r, s), \int_{\mathbb{R}} U_{\gamma_{t}}(r, s, z) v(d z)\right)-h\left(s, \bar{Z}_{\gamma_{t}}(r, s), \int_{\mathbb{R}} \bar{U}_{\gamma_{t}}(r, s, z) v(d z)\right) \\
& =\int_{0}^{1} \frac{\partial}{\partial z} h\left(g^{s}(\theta)\right) d \theta \cdot\left(Z_{\gamma_{t}}(r, s)-\bar{Z}_{\gamma_{t}, s-}(r, s)\right) \\
& \quad+\int_{0}^{1} \frac{\partial}{\partial u} h\left(g^{s}(\theta)\right) d \theta \cdot \int_{\mathbb{R}} U_{\gamma_{t}}(r, s, z)-\bar{U}_{\gamma_{t}}(r, s, z) v(d z) .
\end{aligned}
$$

Since $\left(Y_{\gamma_{t}}(\cdot), Z_{\gamma_{t}}(\cdot, \cdot), U_{\gamma_{t}}(\cdot, \cdot, \cdot)\right), \quad\left(\bar{Y}_{\gamma_{t}}(\cdot), \bar{Z}_{\gamma_{t}}(\cdot, \cdot), \bar{U}_{\gamma_{t}}(\cdot, \cdot, \cdot)\right)$ are adapted M-solutions to BSVIE (45) corresponding to $(f, h, \Phi)$ and $(\bar{f}, \bar{h}, \bar{\Phi})$, respectively, we get for $s \in[t, T]$ 


$$
\begin{aligned}
& Y_{\gamma_{t}}(s)-\bar{Y}_{\gamma_{t}}(s) \\
& =\hat{\Phi}\left(X_{\gamma_{t}, s}\right)+\int_{s}^{T} A_{0}\left(s, r, X_{\gamma_{t}, r-}\right)\left(Y_{\gamma_{t}}(r-)-\bar{Y}_{\gamma_{t}}(r-)\right)+A_{1}(s)\left(Z_{\gamma_{t}}(r, s)-\bar{Z}_{\gamma_{t}}(r, s)\right) d r \\
& \quad+\int_{s}^{T} \int_{\mathbb{R}} A_{2}(s)\left(U_{\gamma_{t}}(r, s, z)-\bar{U}_{\gamma_{t}}(r, s, z)\right) v(d z) d r-\int_{s}^{T} Z_{\gamma_{t}}(s, r)-\bar{Z}_{\gamma_{t}}(s, r) W(d r) \\
& \quad-\int_{s}^{T} \int_{\mathbb{R}} U_{\gamma_{t}}(s, r, z)-\bar{U}_{\gamma_{t}}(s, r, z) \tilde{N}(d r, d z), \quad s \in[t, T],
\end{aligned}
$$

where

$$
\begin{aligned}
& \hat{\Phi}\left(X_{\gamma_{t}, s}\right) \\
& =\Phi\left(X_{\gamma_{t}, s}\right)-\bar{\Phi}\left(X_{\gamma_{t}, s}\right)+\int_{s}^{T} f\left(s, r, X_{\gamma_{t}, r-}, \bar{Y}_{\gamma_{t}}(r-)\right)-\bar{f}\left(s, r, X_{\gamma_{t}, r-}, \bar{Y}_{\gamma_{t}}(r-)\right) d r \\
& +\int_{s}^{T} h\left(s, \bar{Z}_{\gamma_{t}}(r, s), \int_{\mathbb{R}^{l}} \bar{U}_{\gamma_{t}}(r, s, z) v(d z)\right)-\bar{h}\left(s, \bar{Z}_{\gamma_{t}}(r, s), \int_{\mathbb{R}^{l}} \bar{U}_{\gamma_{t}}(r, s, z) v(d z)\right) d r,
\end{aligned}
$$

and

$$
\begin{aligned}
& A_{0}\left(s, r, X_{\gamma_{t}, r-}\right)=\int_{0}^{1} \frac{\partial}{\partial y} f\left(l_{X_{\gamma_{t}, r-}}^{s, r}(\theta)\right) d \theta, \quad A_{1}(s)=\int_{0}^{1} \frac{\partial}{\partial z} h\left(g^{s}(\theta)\right) d \theta, \\
& A_{2}(s)=\int_{0}^{1} \frac{\partial}{\partial u} h\left(g^{s}(\theta)\right) d \theta .
\end{aligned}
$$

Pick any $\varphi(\cdot) \in L_{\mathbb{F}}^{2}(\Omega \times D([0, T], \mathbb{R}) ; \mathbb{R})$ with $\varphi(\gamma) \geq 0, \gamma \in D([0, T] ; \mathbb{R})$, and consider a path-dependent linear FSVIE of the form

$$
\begin{aligned}
X_{\gamma_{t}}(s)= & \varphi\left(X_{\gamma_{t}, s}\right)+\int_{t}^{s} A_{0}\left(r, s, X_{\gamma_{t}, s-}\right) X_{\gamma_{t}}(r-) d r+\int_{t}^{s} A_{1}(r) X_{\gamma_{t}}(r-) d W(r) \\
& +\int_{t}^{s} \int_{\mathbb{R}} A_{2}(r) X_{\gamma_{t}}(r-) \tilde{N}(d r, d z),
\end{aligned}
$$

for $s \in[t, T]$. Theorem 6 yields $X_{\gamma_{t}}(s) \geq 0$ a.s. Moreover, it is $\hat{\Phi}\left(X_{\gamma_{t}, s}\right) \geq 0$, a.s. The duality principle (Theorem 5) implies for $s \in[t, T]$

$\mathbb{E}\left[\int_{t}^{T}\left\langle\varphi\left(X_{\gamma_{t}, u-}\right), Y_{\gamma_{t}}(u-)-\bar{Y}_{\gamma_{t}}(u-)\right\rangle d u\right]=\mathbb{E}\left[\int_{t}^{T}\left\langle X_{\gamma_{t}}(u-), \hat{\Phi}\left(X_{\gamma_{t}, u-}\right)\right\rangle d u\right] \geq 0$ and we obtain

$$
\mathbb{E}\left[\int_{t}^{T} Y_{\gamma_{t}}(u-) d u\right] \geq \mathbb{E}\left[\int_{t}^{T} \bar{Y}_{\gamma_{t}}(u-) d u\right]
$$

\section{Path dependent BSVIEs and dynamic risk measures}

As indicated in the introduction, BSVIEs are related to dynamic risk measures, see Yong (2007). If the risk measures are now based on path-dependent BSVIEs, the risk measures exhibit an additional dependence from an underlying process $X$. This 
process can be called a factor process or asset price process and may drive the value process $\Phi=\Phi(X)$ as well as the generator of the BSVIE.

Then, differentiability enables the understanding of the sensitivity of the risk measure with respect to the underlying process $X$.

With the help of the comparison theorem, we are able to define a class of continuous-time dynamic risk measures by the solution of a path-dependent BSVIE with jumps. The following two definitions are taken from Yong (2007), which we modify for our purpose. Since the results of the previous sections can be applied, the proofs from Yong (2007) can also be modified.

Definition 3 A map $\rho: L_{\mathcal{F}_{T}}^{2}(t, T) \rightarrow L_{\mathbb{F}}^{2}(t, T)$ is called a dynamic risk measure if the following hold

1. (Past Independence) For any $\Phi(\cdot), \bar{\Phi}(\cdot) \in L_{\mathcal{F}_{T}^{t}}^{2}\left(D\left([0, T], \mathbb{R}^{d}\right) ; \mathbb{R}\right)$, if

$$
\Phi\left(\gamma_{s}\right)=\bar{\Phi}\left(\gamma_{s}\right), \quad \text { a.s. } \omega \in \Omega, s \in[r, T],
$$

for some $r \in[t, T]$, then

$$
\rho(r ; \Phi(\cdot))=\rho(r ; \bar{\Phi}(\cdot)), \quad \text { a.s. } \omega \in \Omega .
$$

2. (Monotonicity) For any $\Phi(\cdot), \bar{\Phi}(\cdot) \in L_{\mathcal{F}_{T}^{t}}^{2}\left(D\left([0, T], \mathbb{R}^{d}\right) ; \mathbb{R}\right)$, if

$$
\Phi(\gamma) \leq \bar{\Phi}(\gamma), \quad \text { a.s. } \omega \in \Omega, \gamma \in D\left([0, T], \mathbb{R}^{d}\right)
$$

then

$$
\rho(s ; \Phi(\cdot)) \geq \rho(s ; \bar{\Phi}(\cdot)), \quad \text { a.s. } \omega \in \Omega, s \in[t, T] .
$$

Definition 4 A dynamic risk measure $\rho: L_{\mathcal{F}_{T}}^{2}(t, T) \rightarrow L_{\mathbb{F}}^{2}(t, T)$ is called a dynamic coherent risk measure if the following hold

1. (Positive homogeneity) For any $\Phi(\cdot) \in L_{\mathcal{F}_{T}^{t}}^{2}\left(D\left([0, T], \mathbb{R}^{d}\right) ; \mathbb{R}\right)$ and $\lambda \in \mathbb{R}^{+}$,

$$
\rho(s ; \lambda \Phi(\cdot))=\lambda \cdot \rho(s ; \Phi(\cdot)), \quad \text { a.s. } \omega \in \Omega, s \in[t, T] .
$$

2. (Sub-additivity) For any $\Phi(\cdot), \bar{\Phi}(\cdot) \in L_{\mathcal{F}_{T}^{t}}^{2}\left(D\left([0, T], \mathbb{R}^{d}\right) ; \mathbb{R}\right)$

$$
\rho(s ; \Phi(\cdot)+\bar{\Phi}(\cdot)) \leq \rho(s ; \Phi(\cdot))+\rho(s ; \bar{\Phi}(\cdot)), \quad \text { a.s. } \omega \in \Omega, s \in[t, T] .
$$

3. (Translation invariance) There exists a deterministic integrable function $r(\cdot, \cdot)$ such that for any $\Phi(\cdot) \in L_{\mathcal{F}_{T}^{t}}^{2}\left(D\left([0, T], \mathbb{R}^{d}\right) ; \mathbb{R}\right)$

$$
\rho(s ; \Phi(\cdot)+c)=\rho(s ; \Phi(\cdot))-c e^{-\int_{s}^{T} r(s, u) d u}, \quad \text { a.s. } \omega \in \Omega, s \in[t, T] .
$$

We denote

$$
\rho(s, \Phi(\cdot))=\mathbb{E}\left[\int_{t}^{T} Y_{\gamma_{t}}(s-) d s\right],
$$


where $\left(Y_{\gamma_{t}}(\cdot), Z_{\gamma_{t}}(\cdot, \cdot), U_{\gamma_{t}}(\cdot, \cdot, \cdot)\right)$ is the unique adapted M-solution to the following path-dependent BSVIE with jumps

$$
\begin{aligned}
& Y_{\gamma_{t}}(s) \\
& =-\Phi\left(X_{\gamma_{t}, s}\right)+\int_{s}^{T} f\left(s, r, X_{\gamma_{t}, r-}, Y_{\gamma_{t}}(r-)\right)+h\left(s, Z_{\gamma_{t}}(r, s), \int_{\mathbb{R}^{l}} U_{\gamma_{t}}(r, s, z) v(d z)\right) d r \\
& \quad-\int_{s}^{T} Z_{\gamma_{t}}(s, r) d W(r)-\int_{s}^{T} \int_{\mathbb{R}^{l}} U_{\gamma_{t}}(s, r, z) \tilde{N}(d r, d z), \quad s \in[t, T] .
\end{aligned}
$$

Proposition 1 1. Let f and h satisfy (H2) and (H3). Moreover, suppose that both are sub-additive, i.e.,

$$
\begin{aligned}
f\left(s, r, \gamma, y_{1}+y_{2}\right) & \leq f\left(s, r, \gamma, y_{1}\right)+f\left(s, r, \gamma, y_{2}\right) \\
h\left(s, z_{1}+z_{2}, u_{1}+u_{2}\right) & \leq h\left(s, z_{1}, u_{1}\right)+h\left(s, z_{2}, u_{2}\right)
\end{aligned}
$$

for all $(s, r) \in[t, T]^{2}, y_{1}, y_{2} \in \mathbb{R}, z_{1}, z_{2} \in \mathbb{R}^{d}, u_{1}, u_{2} \in \mathbb{R}$. Then, $\Phi(\cdot) \mapsto$ $\rho(s ; \Phi(\cdot))$ is sub-additive, i.e.,

$$
\rho\left(s ; \Phi_{1}(\cdot)+\Phi_{2}(\cdot)\right) \leq \rho\left(s ; \Phi_{1}(\cdot)\right)+\rho\left(s ; \Phi_{2}(\cdot)\right) \text {, a.e. }
$$

2. If $f:[t, T]^{2} \times D\left([0, T], \mathbb{R}^{d}\right) \times \mathbb{R} \rightarrow \mathbb{R}$ and $h:[t, T] \times \mathbb{R}^{d} \times \mathbb{R} \rightarrow \mathbb{R}$ are positively homogeneous, i.e.,

$$
f(s, r, \gamma, \lambda y)=\lambda \cdot f(s, r, \gamma, y), \quad h(s, \lambda z, \lambda u)=\lambda \cdot h(s, z, u),
$$

for any $\lambda \in \mathbb{R}^{+},(s, r) \in[t, T]^{2}, \gamma \in D\left([0, T], \mathbb{R}^{d}\right), y \in \mathbb{R}, z \in \mathbb{R}^{d}$ and $u \in \mathbb{R}$ a.e., then $\Phi(\cdot) \mapsto \rho(s, \Phi(\cdot))$ is also positively homogeneous.

3. If $f$ is independent of $\gamma \in D\left([0, T], \mathbb{R}^{d}\right)$ and of the form $f(s, r, y)=r(s, r) y$ with a deterministic integrable function $r(\cdot, \cdot)$, then $\Phi(\cdot) \rightarrow \rho(s ; \Phi(\cdot))$ is transition invariant, i.e.,

$\rho(s ; \Phi(\cdot)+c)=\rho(s ; \Phi(\cdot))-e^{\int_{s}^{T} r(s, u) d u}, \quad$ a.s. $\omega \in \Omega, s \in[t, T]$, for allc $\in \mathbb{R}$.

In particular, if $f(s, r, \gamma, y)=0$, then $\rho(s, \Phi(\cdot)+c)=\rho(s, \Phi(\cdot))-c$ a.s. for any $c \in \mathbb{R}$.

Theorem 8 Suppose $f$ and $h$ satisfy (H2) and (H3). Moreover, $f(s, r, \gamma, y)=$ $r(s, r) y$, with $r(\cdot, \cdot)$ being a bounded and deterministic integrable function. Then, $\rho(\cdot)$, defined by (48), is a dynamic coherent risk measure if $f$ and $h$ are positively homogeneous and sub-additive.

Proof The proof is given by Proposition 1 and the comparison theorem for pathdependent BSVIEs with jumps (Theorem 7).

Acknowledgements The authors thank the editor and two anonymous referees for their helpful suggestions.

\section{Authors' contributions}

Both authors read and approved the final manuscript. 


\section{Competing interests}

The authors declare that they have no competing interests.

\section{References}

Aman, A, N'Zi, M: Backward stochastic nonlinear Volterra integral equations with local Lipschitz drift. Probab. Math. Stat. 25, 105-127 (2005)

Anh, V, Yong, J. In: Agarwal, RP, Perera, K (eds.): Backward stochastic Volterra integral equations in Hilbert spaces, Differential and Difference Equations and Applications, pp. 57-66. Hindawi Publishing Corporation (2006). http://scholar.google.de/scholar_url?url=http://downloads.hindawi.com /books/9789775945389.pdf\%23page\%3D72\&hl=de\&sa=X\&scisig=AAGBfm1iiQIdscsJGWvzJgRjh4 \&nossl=1\&oi=scholarr\&ved=0ahUKEwjL7OOu8ebaAhSOa1JdDwVkAcAKHfkqDS8QgAMILSgAMAA

Ankirchner, S, Imkeller, P, Dos Reis, G: Classical and variational differentiability of BSDEs with quadratic growth. Electron. J. Probab. 12, 1418-1453 (2007)

Barles, G, Buckdahn, R, Pardoux, E: Backward stochastic differential equations and integral-partial differential equations. Stochastics Stochastics Rep. 60, 57-83 (1997)

Becherer, D: Bounded solutions to bsdes with jumps for utility optimization and indifference hedging. Ann. Appl. Probab. 16, 2027-2054 (2006)

Berger, M, Mizel, V: Volterra equations with itô integrals. J Integr. Equ. 2, 187-245 (1980)

Carmona, R: Indifference Pricing: Theory and Applications. Princeton University Press, Princeton (2008)

Cont, R, Fournié, DA: Change of variable formulas for non-anticipative functionals on path space. J. Funct. Anal. 259, 1043-1072 (2010)

Crépey, S: Financial Modeling, a backward stochastic differential equations perspective. Springer-Verlag Berlin Heidelberg (2013)

Delong, L: Backward stochastic differential equations with jumps and their actuarial and financial applications. Springer-Verlag London, London (2013)

Doléans-Dade, C: Quelques applications de la formule de changement de variables pour les semimartingales. Z Wahrscheinlichkeitstheorie verw Gebiete. 16, 181-194 (1970)

Duffie, D, Epstein, LG: Stochastic differential utility. Econometrica. 60(2), 353-394 (1992)

Dupire, B: Functional Itô calculus (2009). Bloomberg Portfolio Research Paper No. 2009-04FRONTIERS. Available at SSRN: https://ssrn.com/abstract=1435551

El Karoui, N, Peng, S, Quenez, M: Backward stochastic differential equations in finance. Math. Financ. 7, 1-71 (1997)

He, S, Wang, J, Yan, J: Semimartingale Theory and Stochastic Calculus. CRC Press, Boca Raton (1992)

Jacod, J, Shiryaev, AN: Limit theorems for stochastic processes, 2nd edn. Springer-Verlag Berlin Heidelberg, Berlin (2003)

Kromer, E, Overbeck, L: Representation of BSDE-based dynamic risk measures and dynamic capital allocations. Int. J. Theor. Appl. Financ. 17, 1-16 (2014)

Kromer, E, Overbeck, L: Differentiability of BSVIEs and dynamic capital allocations. Int. J. Theor. Appl. Financ. 20, 1-26 (2017)

Kromer, E, Overbeck, L, Röder, J: Feynman Kac for functional jump diffusions with an application to credit value adjustment. Stat. Probab. Lett. 105, 120-129 (2015)

Kromer, E, Overbeck, L, Röder, J: Path-dependent BSDEs with jumps and their connection to PPIDEs. Stochast. Dyn. 17, 1-37 (2017)

Lazrak, A, Quenez, M: A generalized stochastic differential utility. Math. Oper. Res. 28, 154-180 (2003)

Levental, S, Schroder, M, Sinha, S: A simple proof of functional Itô's lemma for semimartingales with an application. Stat. Probab. Lett. 83, 2019-2026 (2013)

Lin, J: Adapted solution of a backward stochastic nonlinear Volterra integral equation. Stoch. Anal. Appl. 20, 165-183 (2002)

Lu, W: Backward stochastic Volterra integral equations associated with a Lévy process and applications (2016). Available at arXiv: https://arxiv.org/abs/1106.6129

Pardoux, E, Peng, S: Adapted solution of a backward stochastic differential equation. Syst. Control Lett. 14, 55-61 (1990)

Pardoux, E, Protter, P: Stochastic Volterra equations with anticipating coefficients. Ann. Probab. 18, 16351655 (1990) 
Peng, S, Wang, F: BSDE, path-dependent PDE and nonlinear Feynman-Kac formula. Sci. China Math. 59, 1-18 (2016)

Protter, P: Volterra equations driven by semimartingales. Ann. Probab. 13, 519-530 (1985)

Ren, Y: On solutions of backward stochastic volterra integral equations with jumps in hilbert spaces. J. Optim. Theory Appl. 144, 319-333 (2010)

Rong, S: On solutions of backward stochastic differential equations with jumps and applications. Stoch. Process. Appl. 66, 209-236 (1997)

Tang, S, Li, X: Necessary conditions for optimal control of stochastic systems with random jumps. SIAM J. Control. Optim. 32, 1447-1475 (1994). https://doi.org/101137/S0363012992233858

Wang, F: BSDEs with jumps and path-dependent parabolic integro-differential equations. Chin. Ann. Math. Ser. B. 36, 625-644 (2015)

Wang, T, Yong, J: Comparison theorems for some backward stochastic Volterra integral equations. Stoch. Process. Appl. 125, 1756-1798 (2015)

Wang, Z, Zhang, X: Non-Lipschitz backward stochastic Volterra type equations with jumps. Stochastics and Dynamics. 7(4), 479-496 (2007)

Yong, J: Backward stochastic Volterra integral equations and some related problems. Stoch. Process. Appl. 116, 779-795 (2006)

Yong, J: Continuous-time dynamic risk measures by backward stochastic Volterra integral equations. Appl Anal. 186, 1429-1442 (2007)

Yong, J: Well-posedness and regularity of backward stochastic volterra integral equations. Probab. Theory Relat. Fields. 142, 21-77 (2008) 